\title{
Coal Quality and Major, Minor, and Trace Elements in the Powder River, Green River, and Williston Basins, Wyoming and North Dakota
}

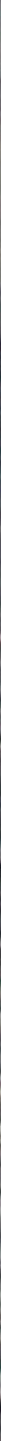




\section{Coal Quality and Major, Minor, and Trace Elements in the Powder River, Green River, and Williston Basins, Wyoming and North Dakota}

By Gary D. Stricker, Romeo M. Flores, Michael H. Trippi, Margaret S. Ellis, Carol M. Olson, Jonah E. Sullivan, and Kenneth I. Takahashi

Open-File Report 2007-1116 


\title{
U.S. Department of the Interior DIRK KEMPTHORNE, Secretary
}

\author{
U.S. Geological Survey \\ Mark D. Myers, Director
}

U.S. Geological Survey, Reston, Virginia: 2007

For product and ordering information:

World Wide Web: http://www.usgs.gov/pubprod

Telephone: 1-888-ASK-USGS

For more information on the USGS - the Federal source for science about the Earth, its natural and living resources, natural hazards, and the environment:

World Wide Web: http://www.usgs.gov

Telephone: 1-888-ASK-USGS

Any use of trade, product, or firm names is for descriptive purposes only and does not imply endorsement by the U.S. Government.

Although this report is in the public domain, permission must be secured from the individual copyright owners to reproduce any copyrighted materials contained within this report.

Suggested citation:

Stricker, G.D., Flores, R.M., Trippi, M.H., Ellis, M.S., Olson, C.M., Sullivan, J.E., and Takahashi, K.I., 2007, Coal quality and major, minor, and trace elements in the Powder River, Green River, and Williston basins, Wyoming and North Dakota: U.S. Geological Survey Open-File Report 2007-1116 


\section{Contents}

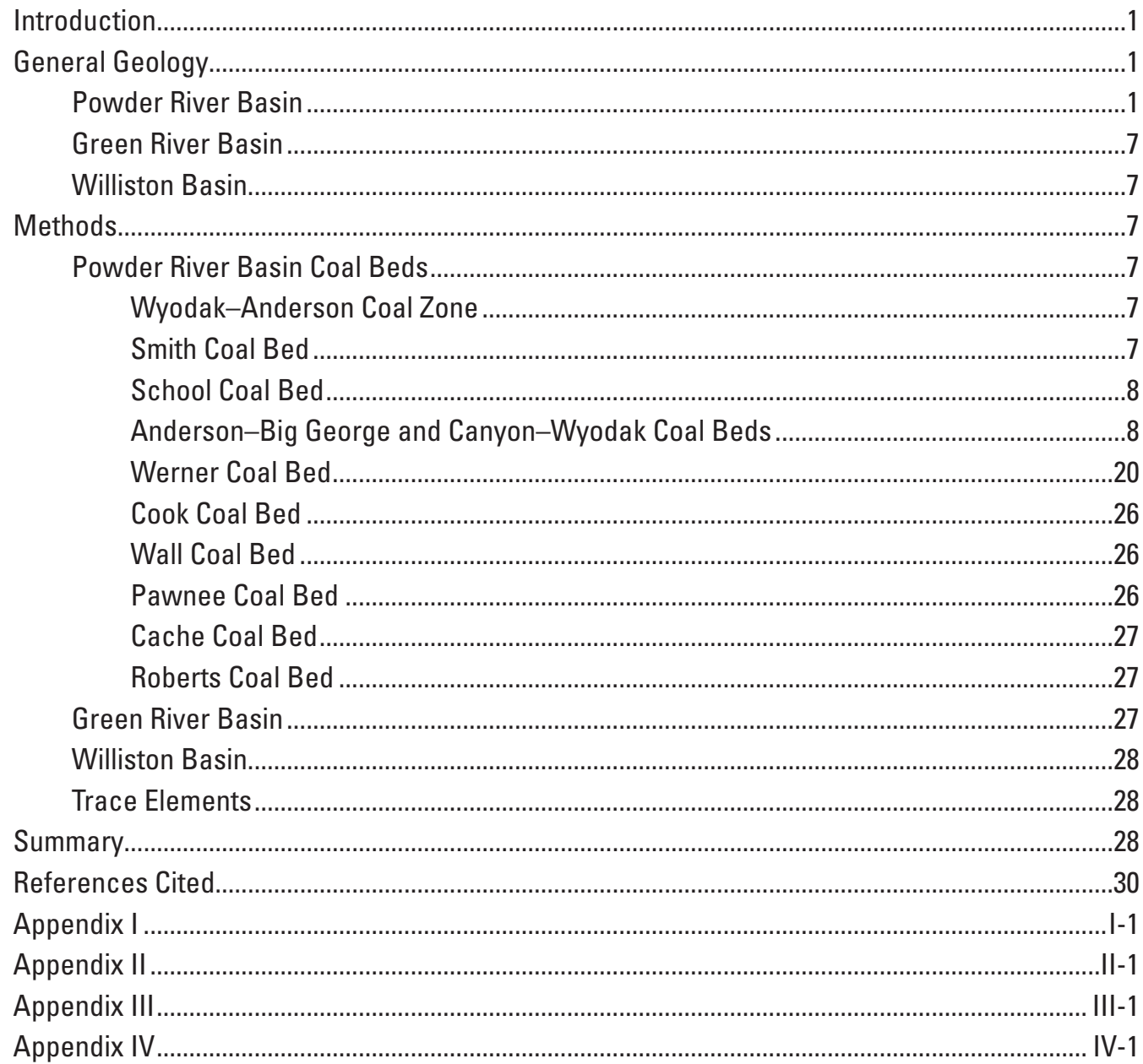

\section{Figures}

1. Location of core holes in the Powder River and Green River Basins, Wyoming and Williston Basin, North Dakota

2. Stratigraphic column for the Fort Union Formation in the Powder River Basin, Wyoming and Montana

3. Coal quality data for coal beds in the Fort Union Formation in the Powder River Basin, Wyoming

4. Stratigraphic column for the Fort Union Formation in the Green River Basin, Wyoming

5. Stratigraphic column for the Fort Union Formation in the Williston Basin, North Dakota

6. Variation of moist, mineral-matter-free (MMMF) British thermal units (Btu) in the Wyodak-Anderson coal zone in the Powder River Basin, Wyoming 
7. Variation of moisture content in the Wyodak-Anderson coal zone in the Powder River Basin, Wyoming .........................................................................................22

8. Variation of ash yield in the Wyodak-Anderson coal zone in the Powder River Basin, Wyoming

9. Variation of sulfur content in the Wyodak-Anderson coal zone in the Powder River Basin, Wyoming ...............................................................................................24

10. Ash partings in the merged Wyodak coal bed, eastern Powder River Basin, Wyoming.

\section{Tables}

1. List of core holes used in coal studies, Powder River, Green River, and Williston Basins, Wyoming and North Dakota.

2. Properties and composition of coal beds cored in the Powder River, Green River, and Williston Basins, Wyoming and North Dakota ...

3. Coal quality for coal beds in the Fort Union Formation in the Powder River Basin, Wyoming

4. Major, minor, and the trace elements in the Wyodak and Big George coal beds, Powder River Basin, Wyoming 


\title{
Coal Quality and Major, Minor, and Trace Elements in the Powder River, Green River, and Williston Basins, Wyoming and North Dakota
}

\author{
By Gary D. Stricker ${ }^{1}$, Romeo M. Flores ${ }^{1}$, Michael H. Trippi², Margaret S. Ellis ${ }^{1}$, Carol M. Olson', \\ Jonah E. Sullivan', and Kenneth I. Takahashi
}

\section{Introduction}

The U.S. Geological Survey (USGS), in cooperation with the Wyoming Reservoir Management Group (RMG) of the Bureau of Land Management (BLM) and nineteen independent coalbed methane (CBM) gas operators in the Powder River and Green River Basins in Wyoming and the Williston Basin in North Dakota, collected 963 coal samples from 37 core holes

(fig. 1; table 1) between 1999 and 2005. The drilling and coring program was in response to the rapid development of CBM, particularly in the Powder River Basin (PRB), and the needs of the RMG BLM for new and more reliable data for CBM resource estimates and reservoir characterization. The USGS and BLM entered into agreements with the gas operators to drill and core Fort Union coal beds, thus supplying core samples for the USGS to analyze and provide the RMG with rapid, real-time results of total gas desorbed, coal quality, and high pressure methane adsorption isotherm data (Stricker and others, 2006).

The USGS determined the ultimate composition of all coal core samples; for selected samples analyses also included proximate analysis, calorific value, equilibrium moisture, apparent specific gravity, and forms of sulfur. Analytical procedures followed those of the American Society of Testing Materials (ASTM; 1998). In addition, samples from three wells (129 samples) were analyzed for major, minor, and trace element contents. Ultimate and proximate compositions, calorific value, and forms of sulfur are fundamental parameters in evaluating the economic value of a coal. Determining trace element concentrations, along with total sulfur and ash yield, is also essential to assess the environmental effects of coal use, as is the suitability of the coal for cleaning, gasification, liquefaction, and other treatments. Determination of coal quality in the deeper part (depths greater than 1,000 to 1,200 ft) of the PRB (Rohrbacher and others, 2006; Luppens and others, 2006) is especially important, because these coals are targeted for future mining and development.

${ }^{1}$ U.S. Geological Survey, Denver, Colorado 80225

${ }^{2}$ U.S. Geological Survey, Reston, Virginia 20192
This report contains summary tables, histograms, and isopleth maps of coal analyses. Details of the compositional internal variability of the coal beds are based on the continuous vertical sampling of coal sequences, including beds in the deeper part of the PRB. Such sampling allows for close comparisons of the compositions of different parts of coal beds as well as within the same coal beds at different core hole locations within short distances of each other.

\section{General Geology}

\section{Powder River Basin}

Coal samples collected in the PRB targeted thick coal beds and coal zones that are producing CBM or have potential for CBM production. The sampled coal beds and coal zones are in the Tongue River Member of the Paleocene Fort Union Formation; they include, in ascending order, the Roberts, Cache, Pawnee, Wall, and Cook coal beds and beds in the Wyodak-Anderson coal zone. The later is comprised of the Werner, Canyon-Wyodak, Anderson-Big George, School, Badger, and Smith coal beds (fig. 2), with individual beds ranging in thickness from 20 to $205 \mathrm{ft}$. The zone extends laterally about 190 mi north-south and 63 mi east-west (Flores and others, 1999c). Within it, beds split and merge, and several are the most widespread in the PRB.

Names of the sampled coal beds used in this study are those given by gas operations, and do not necessarily follow USGS names and correlations as established in earlier studies by Mapel (1973), Kent (1986), Culbertson and others (1979), and Flores and others (1999c). Figure 3 shows some of the inconsistencies that have arisen in the naming and correlation of the Cook, Canyon, and Anderson coal beds by gas operators in two different but adjacent lease areas of the PRB (Flores and others, 2005). Because of these inconsistencies, discussions and comparisons of coal quality and major, minor, and trace element data are limited to coal beds of the Wyodak-Anderson coal zone (Anderson-Big George and Canyon-Wyodak) for which stratigraphic positions and correlations are considered to be less problematic (see Flores and others, 1999c; McGarry and Flores, 2004). 


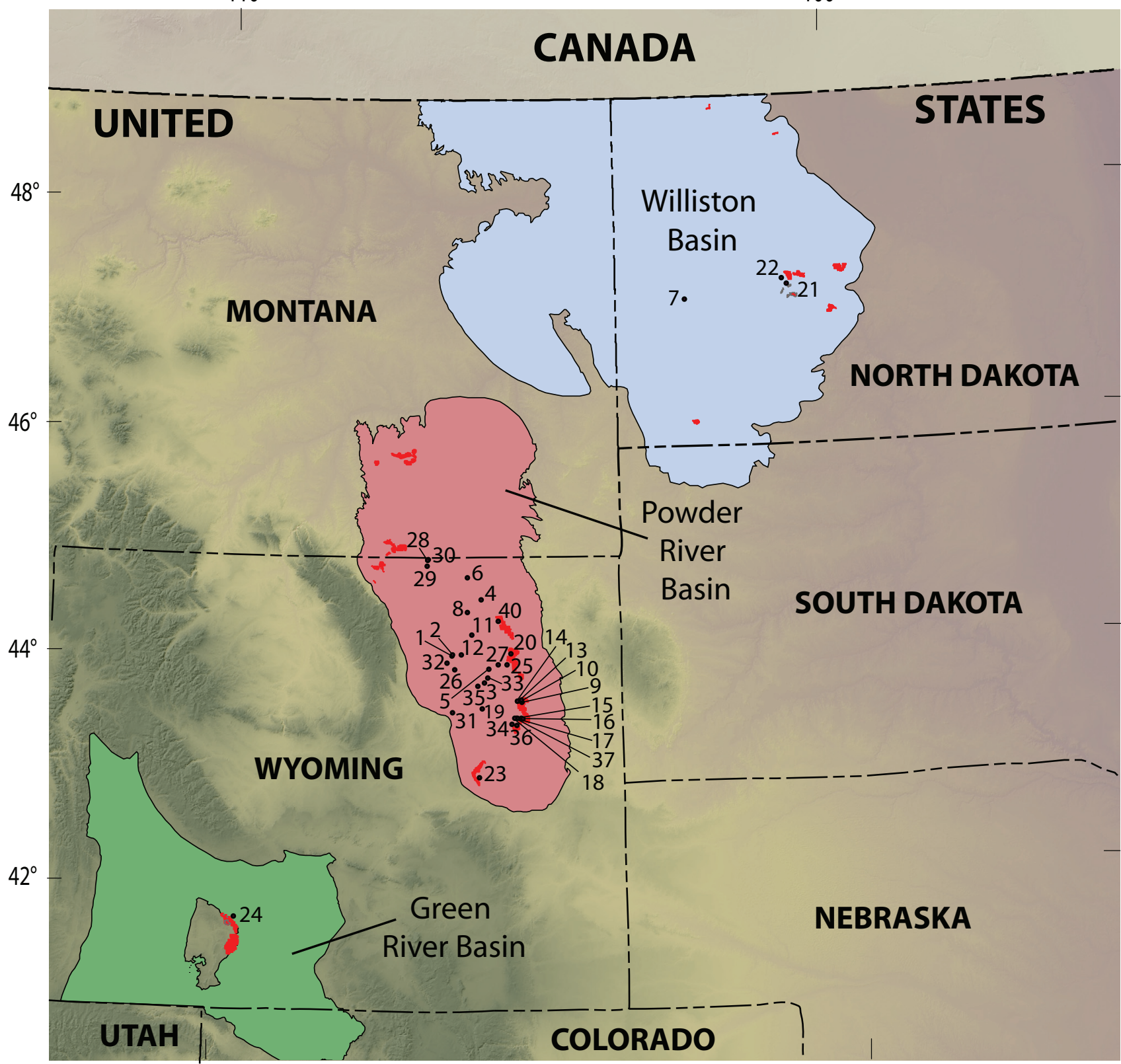

\section{EXPLANATION}

-4 Numbered core hole

- Surface coal mine

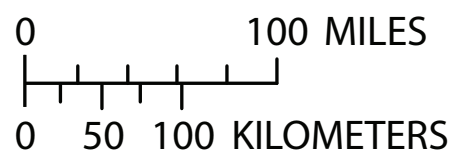

Figure 1. Location of core holes in the Powder River and Green River Basins, Wyoming and Williston Basin, North Dakota. 
Table 1. 3

Table 1. List of core holes used in coal studies, Powder River, Green River, and Williston Basins, Wyoming and North Dakota.

[WY, Wyoming; ND, North Dakota; PRB, Powder River Basin; WB, Williston Basin; GRB, Green River Basin; NA, no API number]

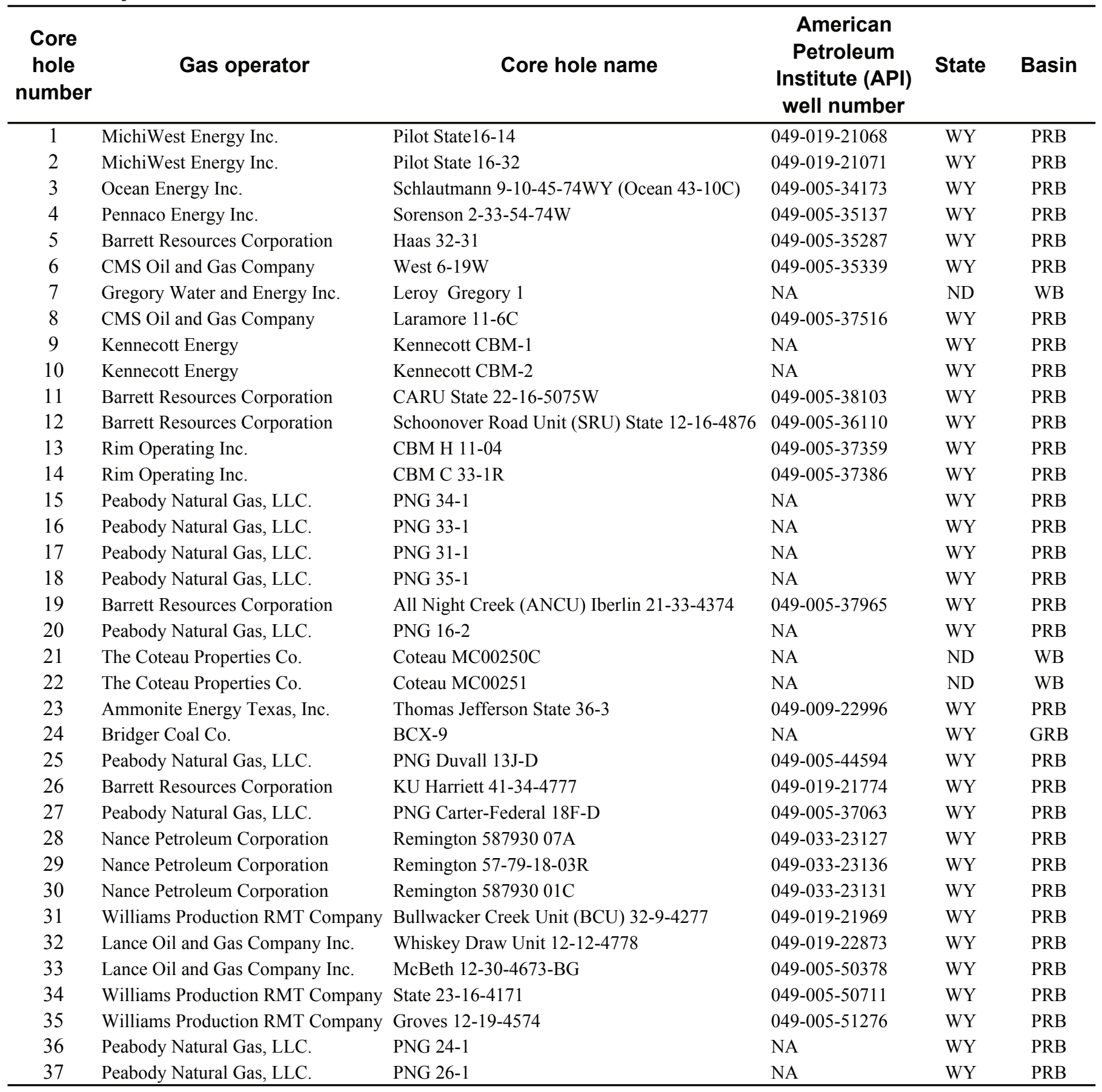


Table 1. List of core holes used in coal studies - Continued

\begin{tabular}{|c|c|c|c|c|}
\hline $\begin{array}{c}\text { Core } \\
\text { hole } \\
\text { number }\end{array}$ & $\begin{array}{l}\text { Decimal } \\
\text { latitude }\end{array}$ & $\begin{array}{c}\text { Decimal } \\
\text { longitude }\end{array}$ & Section, Township, and Range & Date cored \\
\hline 1 & $44.12838 \mathrm{~N}$ & $106.12824 \mathrm{~W}$ & SW/4 SW/4 Sec 16 T48N R77W & January 27, 1999 \\
\hline 2 & $44.13568 \mathrm{~N}$ & $106.11822 \mathrm{~W}$ & $\mathrm{SW} / 4 \mathrm{NE} / 4 \mathrm{Sec} 16 \mathrm{~T} 48 \mathrm{~N}$ R77W & April 7, 1999 \\
\hline 3 & $43.88730 \mathrm{~N}$ & $105.73104 \mathrm{~W}$ & NE/4 SE/4 Sec 10 T45N R74W & June 25, 1999 \\
\hline 4 & $44.62333 \mathrm{~N}$ & $105.76500 \mathrm{~W}$ & NW/4 NE/4 Sec 33 T54N R74W & August 13, 1999 \\
\hline 5 & $44.00851 \mathrm{~N}$ & $105.67651 \mathrm{~W}$ & SW/4 NE/4 Sec 31 T47N R73W & September 24, 1999 \\
\hline 6 & $44.81796 \mathrm{~N}$ & $105.93621 \mathrm{~W}$ & SE/4 NW/4 Sec 19 T56N R75W & September 27, 1999 \\
\hline 7 & $47.23228 \mathrm{~N}$ & $103.14541 \mathrm{~W}$ & SE/4 SE/4 Sec 6 T143N R98W & October 13, 1999 \\
\hline 8 & $44.51333 \mathrm{~N}$ & $105.93560 \mathrm{~W}$ & NE/4 SW/4 Sec 6 T52N R75W & December 10, 1999 \\
\hline 9 & $43.71728 \mathrm{~N}$ & $105.27447 \mathrm{~W}$ & $\mathrm{SW} / 4 \mathrm{NW} / 4 \mathrm{Sec} 9 \mathrm{~T} 43 \mathrm{~N}$ R70W & January 5,2000 \\
\hline 10 & $43.72136 \mathrm{~N}$ & $105.27680 \mathrm{~W}$ & $\mathrm{SW} / 4 \mathrm{NW} / 4 \mathrm{Sec} 9 \mathrm{~T} 43 \mathrm{~N}$ R70W & January 6,2000 \\
\hline 11 & $44.31003 \mathrm{~N}$ & $105.88602 \mathrm{~W}$ & $\mathrm{SE} / 4 \mathrm{NW} / 4 \mathrm{Sec} 16 \mathrm{~T} 50 \mathrm{~N} 75 \mathrm{~W}$ & February 20, 2000 \\
\hline 12 & $44.13523 \mathrm{~N}$ & $106.00883 \mathrm{~W}$ & $\mathrm{SW} / 4$ NW/4 Sec 16 T48N R76W & February 29, 2000 \\
\hline 13 & $43.73564 \mathrm{~N}$ & $105.27828 \mathrm{~W}$ & NW/4 NW4 Sec 4 T43N R70W & March 22, 2000 \\
\hline 14 & $43.72872 \mathrm{~N}$ & $105.32945 \mathrm{~W}$ & NW/4 SE/4 Sec 1 T43N R71W & April 27, 2000 \\
\hline 15 & $43.57330 \mathrm{~N}$ & $105.26031 \mathrm{~W}$ & NE/4 NW/4 Sec 34 T42N R70W & June 2, 2000 \\
\hline 16 & $43.57583 \mathrm{~N}$ & $105.28145 \mathrm{~W}$ & NE/4 NW/4 Sec 33 T42N R70W & June 2, 2000 \\
\hline 17 & $43.57895 \mathrm{~N}$ & $105.32843 \mathrm{~W}$ & NE/4 NW/4 Sec 31 T42N R70W & June 3, 2000 \\
\hline 18 & $43.57624 \mathrm{~N}$ & $105.35947 \mathrm{~W}$ & $\mathrm{SW} / 4 \mathrm{NE} / 4 \mathrm{Sec} 35 \mathrm{~T} 42 \mathrm{~N}$ R71W & July 12,2000 \\
\hline 19 & $43.66122 \mathrm{~N}$ & $105.75760 \mathrm{~W}$ & NE/4 NW/4 Sec 33 T43N R74W & July 14,2000 \\
\hline 20 & $44.14510 \mathrm{~N}$ & $105.40291 \mathrm{~W}$ & NW/4 NW/4 Sec 9 T48N R71W & July 31, 2000 \\
\hline 21 & $47.33781 \mathrm{~N}$ & $101.82531 \mathrm{~W}$ & C SE/4 NE/4 36 T145N R88W & August 22, 2000 \\
\hline 22 & $47.38478 \mathrm{~N}$ & $101.88874 \mathrm{~W}$ & C NE/4 NE/4 16 T145N R88W & August 24, 2000 \\
\hline 23 & $43.05322 \mathrm{~N}$ & $105.79355 \mathrm{~W}$ & NE/4 NE/4 Sec 36 T36N R75W & September 7, 2000 \\
\hline 24 & $41.80364 \mathrm{~N}$ & $108.70250 \mathrm{~W}$ & $\mathrm{SW} / 4 \mathrm{NW} / 4 \mathrm{Sec} 11 \mathrm{~T} 21 \mathrm{~N}$ R100W & October 24,2000 \\
\hline 25 & $44.04874 \mathrm{~N}$ & $105.45364 \mathrm{~W}$ & NW/4 SE/4 Sec 13 T47N R72W & June 10, 2001 \\
\hline 26 & $44.00798 \mathrm{~N}$ & $106.09271 \mathrm{~W}$ & NE/4 NE/4 Sec 34 T47N R77W & June 13, 2001 \\
\hline 27 & $44.05166 \mathrm{~N}$ & $105.56000 \mathrm{~W}$ & $\mathrm{SE} / 4 \mathrm{NW} / 4 \mathrm{Sec} 18 \mathrm{~T} 47 \mathrm{~N}$ R72W & July 21, 2001 \\
\hline 28 & $44.97265 \mathrm{~N}$ & $106.42874 \mathrm{~W}$ & SW/4 NE/4 Sec 30 T58N R79W & December 5, 2001 \\
\hline 29 & $44.91805 \mathrm{~N}$ & $106.43215 \mathrm{~W}$ & NE/4 NW/4 Sec 18 T57N R79W & December 8, 2001 \\
\hline 30 & $44.97623 \mathrm{~N}$ & $106.42377 \mathrm{~W}$ & NE/4 NE/4 Sec 30 T58N R79W & December 8, 2001 \\
\hline 31 & $43.62830 \mathrm{~N}$ & $106.11680 \mathrm{~W}$ & $\mathrm{SW} / 4 \mathrm{NE} / 4 \mathrm{Sec} 9 \mathrm{~T} 42 \mathrm{~N}$ R77W & January 18, 2002 \\
\hline 32 & $44.06227 \mathrm{~N}$ & $106.18863 \mathrm{~W}$ & SW/4 NW/4 Sec 12 T47N R77W & January 8, 2003 \\
\hline 33 & $43.93458 \mathrm{~N}$ & $105.68611 \mathrm{~W}$ & $\mathrm{SW} / 4 \mathrm{NW} / 4 \mathrm{Sec} 30 \mathrm{~T} 46 \mathrm{~N}$ R73W & April 22, 2003 \\
\hline 34 & $43.52525 \mathrm{~N}$ & $105.39694 \mathrm{~W}$ & NE/4 SW/4 Sec 16 T41N R71W & July 9, 2003 \\
\hline 35 & $43.86103 \mathrm{~N}$ & $105.80693 \mathrm{~W}$ & SW/4 NW/4 Sec 19 T45N R74W & October 22,2003 \\
\hline 36 & $43.51627 \mathrm{~N}$ & $105.33530 \mathrm{~W}$ & $\mathrm{SE} / 4 \mathrm{SW} / 4 \mathrm{Sec} 24 \mathrm{~T} 41 \mathrm{~N}$ R71W & October 25,2003 \\
\hline 37 & $43.57958 \mathrm{~N}$ & $105.34513 \mathrm{~W}$ & $\mathrm{SE} / 4 \mathrm{SE} / 4 \mathrm{Sec} 26 \mathrm{~T} 42 \mathrm{~N}$ R71W & October 28,2003 \\
\hline
\end{tabular}




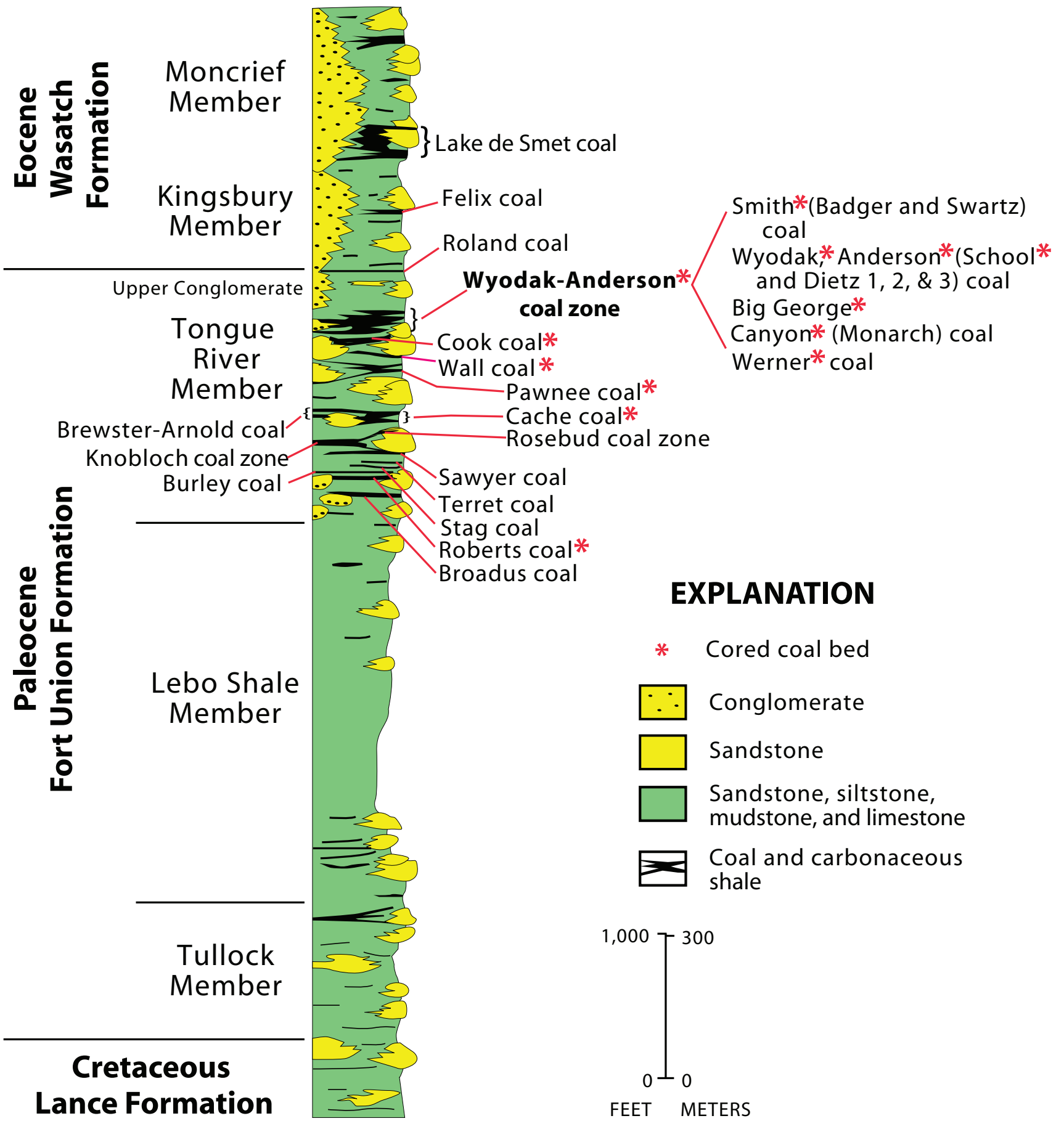

Figure 2. Stratigraphic column for the Fort Union Formation in the Powder River Basin, Wyoming and Montana. 
A

Wolf \#3K-7 Wolf \#2K-11

$\mathrm{TD}=1,030 \mathrm{ft} \quad \mathrm{TD}=983 \mathrm{ft}$

SW NE, Sec. 3 NE SW, Sec. 2

T. $55 \mathrm{~N} ., \quad$ T. $55 \mathrm{~N}$.

D. Odegard Odegard \#6K-5 Odegard

TD $=945 \mathrm{ft} \quad$ \#6K-1

NE SW, Sec. 1 SWNW, Sec. $6 \quad T D=662 \mathrm{ft}$

T. $55 \mathrm{~N}$. $75 \mathrm{~W}$

NE NE, Sec. 6

T. $55 \mathrm{~N}$

R.76 W.

R.75 W.

R.75 W.

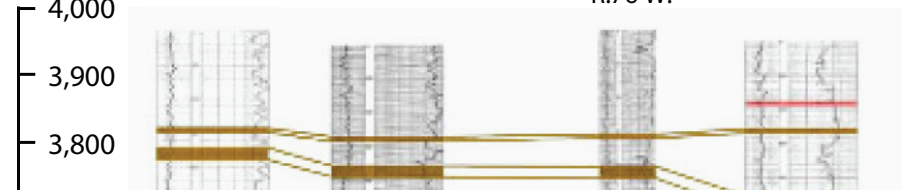

$-3,700$

$-3,600$

$-3,500$

$-3,400$

$-3,300$

$-3,200$

$-3,100$

$-3,000$

- 2,900

- 2,800

$-2,700$

$-2,600$

L,500

ELEVATION

ABOVE MEA

SEA LEVEL,

IN FEET

\section{DEVON LEASE}

\section{EXPLANATION}

Approximate length of cross section is 7 miles

Verticle exaggeration is $15 \mathrm{X}$
$\mathbf{A}^{\prime}$

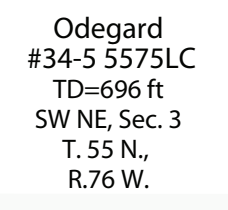

$\begin{array}{cc} & \\ \text { Government \#1 } & \text { Spotted Horse } \\ \text { TD }=8,085 \mathrm{ft} & \text { \#43-4 } 5575 \mathrm{C} \\ \text { NE NW, Sec. } 4 & \text { TD }=530 \mathrm{ft} \\ \text { T. } 55 \text { N., } & \text { NE SE, Sec. } 4 \\ \text { R.75 W. } & \text { T. } 55 \text { N., } \\ & \text { R.74 W. }\end{array}$

SH Odegard

\#34-5575LC

$\mathrm{D}=862 \mathrm{ft}$

SW SE, Sec. 3

T. $55 \mathrm{~N}$

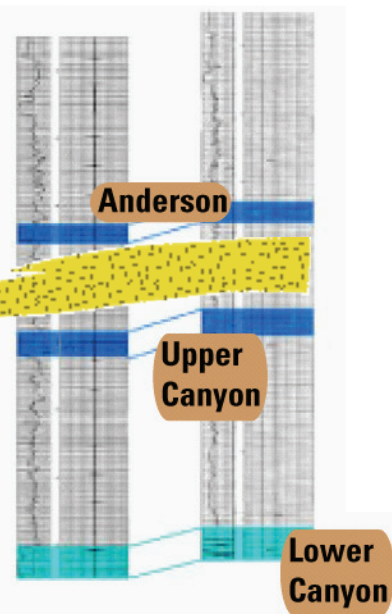

LANCE OIL AND

GAS LEASE

Wyodak-

Anderson

Coal Zone
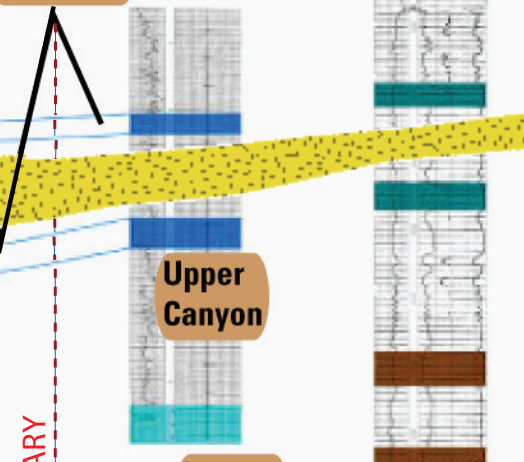

Lower
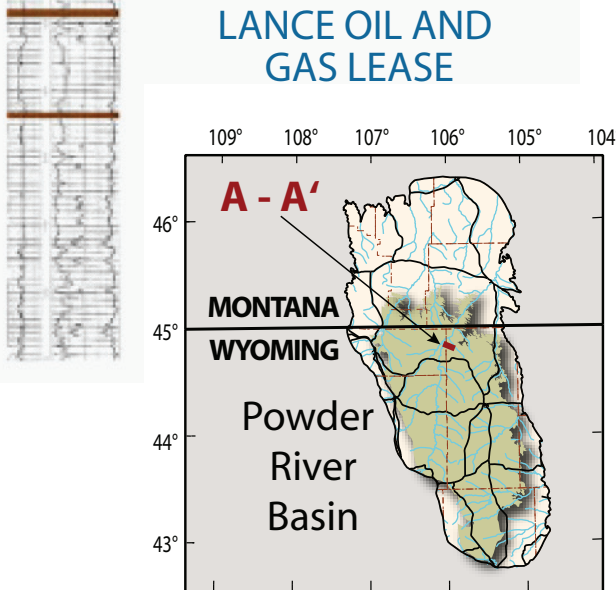

Figure 3. Cross section of coal correlations and coal bed names used by both gas operators and the U.S. Geological Survey for beds in the WyodakAnderson coal zone in the Powder River Basin, Wyoming (coal bed names from Fort Union Assessment Team, 1999; figure modified from Flores and others, 2005). 


\section{Green River Basin}

The Fort Union Formation in the Green River Basin contains two coal zones; Deadman, in the lower part of the Fort Union Formation, and Cherokee, in the upper part. These zones, containing more than 8 individual coal beds (fig. 4), are separated by an interval dominated by fluvial channel sandstones (Hettinger and Kirschbaum, 1991). The sampled coal bed was the Deadman 4 coal (Flores and others, 1999b) in the Bridger Coal Mine (core hole 24 , table 1) where it is as much as $20 \mathrm{ft}$ thick.

\section{Williston Basin}

The Fort Union Formation in the Williston Basin contains 3 coal-bearing intervals: Harmon-Hansen coal zone in the lower part, Hagel coal zone in the middle part, and Beulah-Zap coal zone in the upper part (Flores and others, 1999a); (fig. 5). One coal bed (unnamed 1) sampled in the Leroy 1 well (core hole 7, table 1) is as much as $14 \mathrm{ft}$ thick, and is probably in the Harmon-Hansen zone. Four other coal beds sampled were in the Beulah-Zap coal zone, where they are as much as $50 \mathrm{ft}$ thick. The samples were obtained from the Coteau Coal Mine MC00250C (Beulah and unnamed 2) and MC00251 (unnamed 3 and 4) wells (core holes 21 and 22, respectively; table 1).

\section{Methods}

Coal sampling followed the methods suggested by Stanton (1989), analyses followed the methods of the American Society of Testing Materials (1998), and major-, minor-, and trace-element analyses followed the methods of the USGS (Bullock and others, 2002). The 963 samples that were collected were placed in sealed canisters 2 -ft long and $4.0 \mathrm{in}$. in diameter; and mean sample weight was 3,532 g. The coal, after gas desorption in the canisters (see Stricker and others, 2006), was split into subsamples for (1) ultimate and proximate analysis; (2) determining calorific value, equilibrium moisture value, apparent specific gravity, and forms of sulfur (about $300 \mathrm{~g}$ for (1) and (2)); (3) major-, minor-, and traceelement analysis (about $200 \mathrm{~g}$ ); and (4) archival purposes (about 3,000 g). The samples were put in plastic bags and sealed to minimize moisture loss and possible contamination.

Samples for analyses listed as (1) and (2) above were sent to an outside laboratory (Geochemical Testing) who were also responsible for coal grinding and sample splitting. General procedures are described in the Staff, Office of the Director Coal Research (1967), and specific analyses are described in ASTM (1998). Ultimate analysis included (in percent) ash yield, carbon, hydrogen, oxygen, sulfur, and nitrogen. All of the above analyses are reported and discussed on an as-received basis.

In the USGS laboratory, a sample reserved for major-, minor-, and trace-element analysis was split into two subsamples, one for analysis of whole coal and the other for ashed coal. Elements analyzed on a whole coal basis include chlorine, mercury, total sulfur, and selenium; all other elements were determined on coal ash. Included in the total suite of elements were those of environmental concern (also referred to as hazardous air pollutants or HAPs), which include antimony, arsenic, beryllium, cadmium, chromium, cobalt, lead, manganese, mercury, nickel, selenium, and uranium. For our study, all major-, minor-, and trace-element data analyzed in the USGS laboratory are reported on a whole coal dry basis, as given in Appendix III and shown with bar graphs in Appendix IV.

Results of the ultimate, proximate, calorific value, and forms of sulfur analyses are given in Appendix I, and bar graphs of these data are shown in Appendix II. A statistical summary of data in Appendix I is presented in tables 2 and 3. All analytical data and bar graphs in Appendices I through IV are arranged in ascending order by core hole number. The data will be discussed for selected coal beds according to vertical stratigraphic distribution (in descending order) of the coal beds in each basin.

\section{Powder River Basin Coal Beds}

Twelve different Fort Union Formation coal beds, designated by the names applied by gas operators, were cored and sampled in the PRB (fig. 1). The Wyodak-Anderson coal zone and its contained coal beds Smith, School, Werner, Canyon, Wyodak, Big George, and Anderson and their merged beds (for example, the merged Big George consisting of the combined Anderson and Canyon beds in the west-central part of the PRB, and the merged Wyodak consisting of the combined Anderson and Canyon beds near surface mines in the eastern part of the PRB) contributed the most coal samples (712). This was followed by the Pawnee coal bed (26 samples), Cook coal bed (25 samples), Wall coal bed (20 samples), Cache coal bed (17 samples), Smith coal bed (15 samples), and Roberts coal bed (7 samples). The greatest number of samples were collected from the Wyodak-Anderson coal beds because they are the most productive of CBM in the basin.

\section{Wyodak-Anderson Coal Zone}

Core holes that penetrated the coal beds in the WyodakAnderson coal zone are as follows: Smith, core hole 8; School, core hole 23; Anderson or Big George, core holes 3, 5, 8, 19, 28, 33, 34, and 35; Canyon or Wyodak, core holes 5, 6, 8, 30, and 34; Wyodak (merged Anderson-Canyon or upper, middle, and lower Wyodak), core holes 9, 10, 13-18, and 20; Big George (merged Anderson-Canyon), core holes 1, 2, 11, 12, 26, 31, and 32; and Werner, core hole 11 (see table 2).

\section{Smith Coal Bed}

The Smith coal bed is stratigraphically the highest (youngest) bed cored in the PRB. In core hole 8 (table 1) it consists of the lower and upper Smith beds, which are split by a mudstone in the depth interval 375-363 ft (see core hole 8a, 
Appendix I-13 in Stricker and others, 2006). Listed below are coal properties and composition:

- Apparent rank: subbituminous B

- Calorific value (mean): 9,080 Btu

- Moist-mineral-matter-free Btu (mean): 9,550

- Proximate analysis (mean percent): ash yield, 5.87; volatile matter, 33.08; fixed carbon, 36.46; total moisture, 26.13

- Ultimate analysis (mean percent): hydrogen, 6.22; carbon, 51.27; nitrogen, 0.85; total sulfur, 0.35 ; oxygen, 35.45

- Forms of sulfur (mean percent): sulfate, 0.03; pyritic, 0.04; organic, 0.28

Vertical variations in the proximate and ultimate analyses, calorific value, and forms of sulfur in the lower and upper Smith coal beds are shown in bar graphs for core hole 8 (Appendix II). Moisture in the lower Smith coal bed (375-386 ft) does not vary as much as in the upper Smith coal bed (344-363 ft). Ash yield is higher in the lower Smith coal than in the upper Smith coal. For the combined Smith coal beds, the volatile matter decreases upward and fixed carbon increases upward. Hydrogen, carbon, nitrogen, and oxygen do not show significant variations between the lower and upper Smith coal beds. However, sulfur content shows an increasing upward trend in the lower Smith coal bed and a decreasing upward trend throughout the upper Smith coal bed.

Calorific value is slightly higher in the lower Smith coal bed. Sulfate sulfur is higher in the lower Smith coal bed, whereas pyritic sulfur is highest in the top and bottom of the upper Smith coal bed and in the middle part of the lower Smith coal bed. Organic sulfur increases upward in the lower Smith coal bed but decreases upward in the upper Smith coal bed.

The most distinctive vertical changes in coal quality for the Smith coal bed are ash yield, total sulfur content, and forms of sulfur (Appendix I). The general upward increase in ash yield may be related to the depositional environment, as both the lower and upper Smith coal beds are overlain and separated by floodplain mudstone, which reflects incursions of floodwaters into a low-lying swamp (Pocknall and Flores, 1987; Flores, 1983; 1986) that gradually choked the peatforming environment and caused the upward increase in ash yield now observed in the beds. The relatively high total sulfur content ( 0.35 percent), of the lower Smith coal bed when compared to other coal beds in the Wyodak-Anderson coal zone (Wyodak is 0.18 percent), may be related to the high sulfate and organic sulfur contents.

\section{School Coal Bed}

The School coal bed is in the southwestern part of the PRB. In core hole 23 (table 1), it is subbituminous $C$ in apparent rank with the following properties and composition:
- Calorific value (mean): 8,020 Btu

- Moist-mineral-matter-free Btu (mean): 8,860

- Proximate analysis (mean percent): ash yield, 12.20; volatile matter, 34.11 ; fixed carbon, 30.81 ; total moisture, 25.49

- Ultimate analysis (mean percent): hydrogen, 6.22; carbon, 44.86; nitrogen, 0.65 ; total sulfur, 0.56 ; oxygen, 35.50

- Forms of sulfur (mean percent): sulfate, 0.03; pyritic, 0.11 ; organic, 0.42

Vertical variations exhibited by proximate and ultimate analyses, calorific values, and forms of sulfur in the School coal bed are shown for core hole 23 in Appendices I and II. The ash yield generally decreases upward with a spike (36 percent) in the depth interval 342-343 ft. Total sulfur content increases upward with a spike (1.7 percent) in the depth interval 344-346 ft. The high ash interval corresponds to a parting (see Stricker and others, 2006; core hole 23, Appendix I-33) in the coal directly overlying the high sulfur interval. Pyritic and organic sulfur contents are also correspondingly high in this interval.

The upward decease in ash yield in the School coal bed indicates either a waning flow of floodwaters into the swamp or a gradual raising of the ground level into a raised bog (Flores, 1986). The high ash yield and total sulfur content of this bed were correlated to highly degraded maceral types in it, particularly at the point of splitting, which is an interpretation similar to that given by Moore (1985) for an area of splitting of the Anderson and Dietz coal beds in the Decker area in Montana.

\section{Anderson-Big George and Canyon-Wyodak Coal Beds}

In general, the Anderson-Big George and Canyon-Wyodak coals range from subbituminous $\mathrm{C}$ to $\mathrm{A}$ in apparent rank (see tables 2 and 3). The low rank coal (subbituminous $\mathrm{C}$ ) is located primarily in the shallower part of the basin (surface to 1,000 $\mathrm{ft}$ ), the high rank coal (subbituminous A) is in the deeper part (greater than 1,400 ft), whereas the middle coal rank (subbituminous B) is at an intermediate depth (1,000 to 1,400 ft). Listed below are coal properties and composition:

- Calorific values (range of means): 7,630 to 10,440 Btu

- Moist-mineral-matter-free Btu (range of means): 8,240 to 10,990

- Proximate analysis (range of means in percent): ash yield, 2.92 to 12.22 ; volatile matter, 27.11 to 38.51 ; fixed carbon, 33.18 to 43.79 ; total moisture, 17.95 to 32.01

- Ultimate analysis (range of means in percent): hydrogen, 5.99 to 6.69 ; carbon, 47.41 to 61.46 ; nitrogen, 0.51 to 1.29 ; total sulfur, 0.10 to 1.13 ; oxygen, 27.60 to 39.66

- Forms of sulfur (range of means in percent): sulfate, 0.01 to 0.04 ; pyritic, 0.00 to 0.15 ; organic, 0.11 to 0.56 


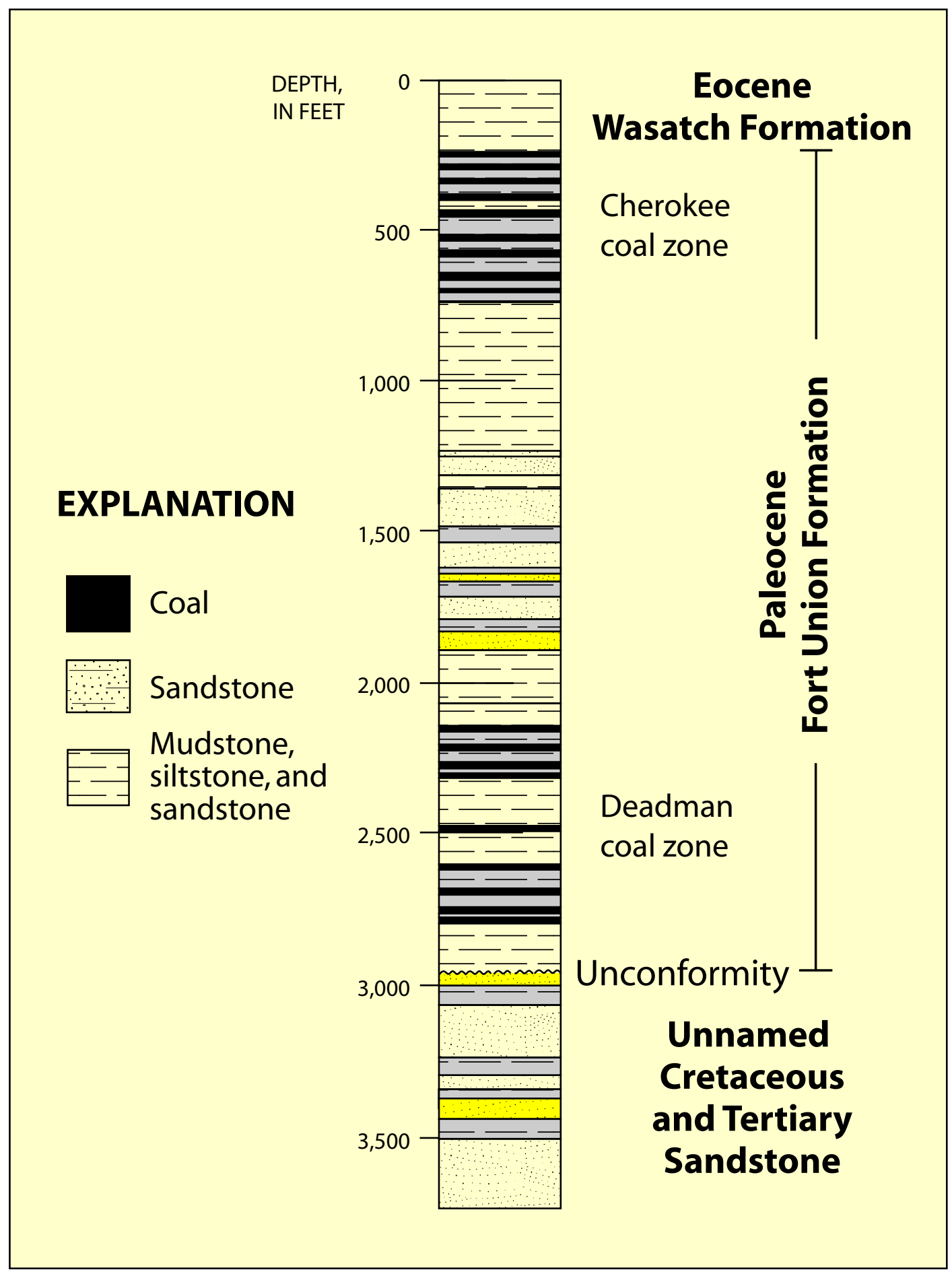

Figure 4. Stratigraphic column for the Fort Union Formation in the Green River Basin, Wyoming. 


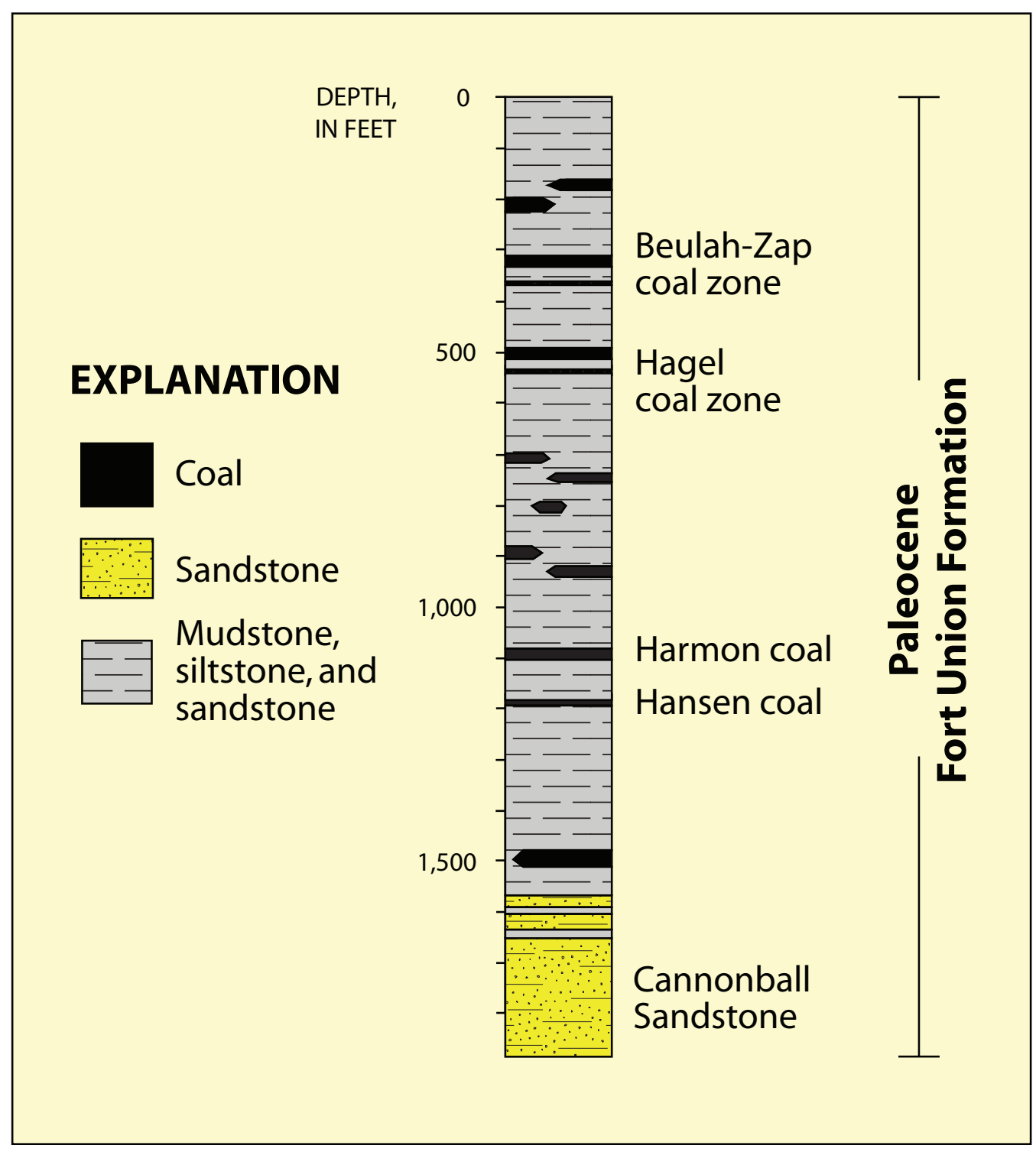

Figure 5. Stratigraphic column for the Fort Union Formation in the Williston Basin, North Dakota. 
Table 2. Properties and composition of coal beds cored in the Powder River, Green River, and Williston Basins, Wyoming and North Dakota.

[----, not analyzed or not applicable; Btu, British thermal units per pound; g/cc, grams per cubic centimeter; LigA, lignite A coal; SubC, subbituminous $C$ coal; SubB, subbituminous B coal; SubA, subbituminous A coal. Values reported on an as-received basis]

\begin{tabular}{|c|c|c|c|c|c|c|c|c|c|c|}
\hline \multirow{2}{*}{$\begin{array}{l}\text { Core } \\
\text { hole }\end{array}$} & \multirow{2}{*}{ Core hole name } & \multirow{2}{*}{$\begin{array}{c}\text { Mean } \\
\text { depth, in } \\
\text { feet }\end{array}$} & \multirow{2}{*}{ Coal zone } & \multirow{2}{*}{$\begin{array}{c}\text { Coal bed name as } \\
\text { used by gas operators }\end{array}$} & \multirow{2}{*}{$\begin{array}{c}\text { Number } \\
\text { of } \\
\text { samples }\end{array}$} & \multicolumn{4}{|c|}{ Moisture, in percent } & \multirow{2}{*}{$\begin{array}{c}\text { Apparent } \\
\text { specific } \\
\text { gravity, in } \\
\text { g/cc }\end{array}$} \\
\hline & & & & & & Residual & $\begin{array}{l}\text { Air dry } \\
\text { loss }\end{array}$ & Total & Equilibrium & \\
\hline 1 & Pilot State 16-14 & $1,287.2$ & Wyodak-Anderson & Big George & 60 & 9.59 & 10.61 & 19.22 & 18.50 & 1.28 \\
\hline 2 & Pilot State 26-32 & $1,138.9$ & Wyodak-Anderson & Big George & 50 & 7.50 & 13.27 & 19.78 & ---- & ---- \\
\hline 3 & Ocean 43-10C & $1,192.0$ & Wyodak-Anderson & Anderson & 2 & 10.32 & 19.09 & 27.76 & 26.40 & ---- \\
\hline 4 & Sorenson 2-33-54-74 & 825.3 & --- & Cook & 10 & 7.53 & 21.42 & 27.30 & ---- & ---- \\
\hline 4 & Sorenson 2-33-54-74 & $1,142.0$ & ---- & Wall & 16 & 9.61 & 17.61 & 25.55 & ---- & ---- \\
\hline 5 & Haas 32-31 2 & $1,076.0$ & Wyodak-Anderson & Big George & 13 & 10.79 & 17.49 & 26.42 & ---- & ---- \\
\hline 5 & Haas 32-31 2 & $1,276.5$ & Wyodak-Anderson & Wyodak & 24 & 9.58 & 16.60 & 24.61 & ---- & ---- \\
\hline 6 & West 6-19 & 648.7 & Wyodak-Anderson & Canyon & 7 & 9.95 & 24.50 & 32.01 & ---- & ---- \\
\hline 6 & West 6-19 & 952.0 & --- & Cook & 8 & 10.87 & 18.80 & 27.77 & ---- & ---- \\
\hline 6 & West 6-19 & $1,074.0$ & ---- & Wall & 4 & 11.92 & 17.58 & 27.40 & ---- & ---- \\
\hline 7 & Leroy 1 & 944.7 & ---- & unnamed 1 & 7 & 13.59 & 20.83 & 31.61 & ---- & ---- \\
\hline 8 & Laramore 11-6 & 385.0 & Wyodak-Anderson & Smith & 15 & 14.50 & 13.58 & 26.13 & ---- & ---- \\
\hline 8 & Laramore 11-6 & 785.2 & Wyodak-Anderson & Anderson & 6 & 7.59 & 19.65 & 25.76 & ---- & ---- \\
\hline 8 & Laramore 11-6 & 990.0 & Wyodak-Anderson & Canyon & 4 & 7.33 & 16.30 & 25.82 & ---- & ---- \\
\hline 8 & Laramore 11-6 & $1,345.0$ & ---- & Cook & 5 & 5.73 & 12.66 & 17.64 & ---- & ---- \\
\hline 9 & Kennecot CBM1 & 143.4 & Wyodak-Anderson & Upper Wyodak & 5 & 4.95 & 15.08 & 19.27 & 22.18 & ---- \\
\hline 9 & Kennecot CBM1 & 181.5 & Wyodak-Anderson & Middle/Lower Wyodak & 26 & 7.27 & 16.62 & 22.69 & 25.57 & ---- \\
\hline 10 & Kennecott CBM2 & 167.0 & Wyodak-Anderson & Upper Wyodak & 2 & 6.96 & 20.93 & 26.46 & 27.45 & 1.26 \\
\hline 10 & Kennecott CBM2 & 209.2 & Wyodak-Anderson & Middle/Lower Wyodak & 27 & 9.43 & 19.92 & 27.53 & 26.78 & 1.28 \\
\hline 11 & Caru State 22-16-5079W & $1,366.4$ & Wyodak-Anderson & Big George & 12 & 8.56 & 17.21 & 24.33 & 23.48 & 1.25 \\
\hline 11 & Caru State 22-16-5079W & $1,769.0$ & Wyodak-Anderson & Werner & 13 & 9.18 & 16.95 & 24.60 & 22.81 & 1.27 \\
\hline 12 & SRU State & $1,401.0$ & Wyodak-Anderson & Big George & 32 & 8.95 & 14.20 & 21.90 & 19.89 & 1.38 \\
\hline 13 & Rim CBM H11-04 & 212.0 & Wyodak-Anderson & Upper Wyodak & 8 & 10.10 & 19.09 & 27.25 & ---- & ---- \\
\hline 13 & Rim CBM H11-04 & 252.4 & Wyodak-Anderson & Middle/Lower Wyodak & 27 & 11.76 & 19.35 & 28.86 & ---- & ---- \\
\hline 14 & Rim 33-1R & 289.0 & Wyodak-Anderson & Middle/Lower Wyodak & 30 & 8.09 & 22.60 & 28.88 & 25.18 & 1.37 \\
\hline 15 & PNG 34-1 & 293.1 & Wyodak-Anderson & Middle/Lower Wyodak & 32 & 9.28 & 20.47 & 27.93 & 25.61 & 1.28 \\
\hline 16 & PNG 33-1 & 319.4 & Wyodak-Anderson & Middle/Lower Wyodak & 28 & 10.53 & 18.50 & 27.13 & 26.13 & 1.30 \\
\hline 17 & PNG 31-1 & 293.1 & Wyodak-Anderson & Middle/Lower Wyodak & 39 & 11.14 & 17.30 & 26.54 & 25.49 & 1.33 \\
\hline 18 & PNG 35-1 & 360.2 & Wyodak-Anderson & Middle/Lower Wyodak & 40 & 12.03 & 15.34 & 25.56 & ---- & ---- \\
\hline 19 & Ancu Iberlin 21-33-4374 & $1,308.0$ & Wyodak-Anderson & Big George & 6 & 14.00 & 11.00 & 23.49 & 23.69 & 1.28 \\
\hline 20 & PNG 16-2 & 248.9 & Wyodak-Anderson & Middle/Lower Wyodak & 34 & 10.49 & 19.33 & 27.81 & 24.77 & ---- \\
\hline
\end{tabular}


Table 2. Properties and composition of coal beds cored-continued

\begin{tabular}{|c|c|c|c|c|c|c|c|c|c|c|}
\hline \multirow{2}{*}{$\begin{array}{l}\text { Core } \\
\text { hole }\end{array}$} & \multirow{2}{*}{ Core hole name } & \multirow{2}{*}{$\begin{array}{l}\text { Average } \\
\text { depth, in } \\
\text { feet }\end{array}$} & \multirow{2}{*}{ Coal zone } & \multirow{2}{*}{$\begin{array}{c}\text { Coal bed name as } \\
\text { used by gas operators }\end{array}$} & \multirow{2}{*}{$\begin{array}{c}\text { Number } \\
\text { of } \\
\text { samples }\end{array}$} & \multicolumn{4}{|c|}{ Moisture, in percent } & \multirow{2}{*}{$\begin{array}{l}\text { Apparent } \\
\text { specific } \\
\text { gravity, in } \\
\text { g/cc }\end{array}$} \\
\hline & & & & & & Residual & $\begin{array}{l}\text { Air dry } \\
\text { loss }\end{array}$ & Total & Equilibrium & \\
\hline 21 & Coteau MC00250C & 172.5 & $\begin{array}{l}--- \\
-1\end{array}$ & Beulah & 2 & 16.51 & 21.41 & 34.41 & $\begin{array}{c}--- \\
\end{array}$ & $\begin{array}{c}--- \\
\end{array}$ \\
\hline 21 & Coteau MC00250C & 442.6 & ---- & unnamed 2 & 4 & 17.05 & 20.34 & 33.93 & ---- & ---- \\
\hline 21 & Coteau MC00250C & 572.2 & ---- & unnamed 3 & 1 & 16.93 & 18.27 & 32.11 & ---- & ---- \\
\hline 22 & Coteau MC00251 & 793.0 & ---- & unnamed 4 & 3 & 13.00 & 21.30 & 31.59 & 29.81 & 1.39 \\
\hline 23 & AET T.J. State $36-3$ & 346.0 & Wyodak-Anderson & School & 9 & 14.07 & 13.29 & 25.49 & 26.25 & 1.35 \\
\hline 24 & BCX-9 (Bridger Coal Co.) & 949.0 & ---- & Deadman 4 & 6 & 6.75 & 10.81 & 16.85 & 17.38 & 1.32 \\
\hline 25 & PNG Duvall 13J-D & $1,243.0$ & ---- & Pawnee & 5 & 7.49 & 19.12 & 25.19 & 24.34 & 1.37 \\
\hline 26 & KU Harriet 41-34-47777 & $1,365.2$ & Wyodak-Anderson & Big George & 51 & 5.29 & 14.60 & 19.23 & 17.67 & 1.31 \\
\hline 27 & PNG Federal Carter 18F & $1,537.6$ & ---- & Pawnee & 11 & 10.04 & 14.87 & 23.43 & 20.41 & 1.30 \\
\hline 27 & PNG Federal Carter 18F & $1,709.8$ & ---- & Cache & 16 & 9.60 & 14.86 & 23.10 & 22.24 & 1.30 \\
\hline 28 & Nance Petroleum Remington 587930 07A & 329.3 & Wyodak-Anderson & Anderson & 8 & 13.70 & 15.93 & 27.46 & 27.23 & 1.31 \\
\hline 29 & Nance Petroleum Remington 577918 03R & $2,197.5$ & ---- & Roberts & 7 & 5.44 & 18.20 & 22.64 & 16.85 & 1.34 \\
\hline 30 & Nance Petroleum Remington 587930 01C & 640.0 & Wyodak-Anderson & Canyon & 7 & 11.05 & 15.98 & 25.31 & 25.53 & 1.31 \\
\hline 31 & BCU Federal 32-9-4277 & $1,440.3$ & Wyodak-Anderson & Big George & 32 & 6.60 & 12.15 & 17.94 & 18.15 & 1.31 \\
\hline 32 & WDU 12-12-4778 & $1,541.8$ & Wyodak-Anderson & Big George & 17 & 7.03 & 11.73 & 17.95 & 16.51 & 1.33 \\
\hline 33 & McBeth-12-30-4673-BG & 960.2 & Wyodak-Anderson & Big George & 10 & 12.96 & 13.56 & 24.77 & 23.83 & 1.29 \\
\hline 34 & State $23-16-4171$ & 345.0 & Wyodak-Anderson & Anderson & 17 & 8.43 & 18.04 & 24.98 & 25.35 & ---- \\
\hline 34 & State $23-16-4171$ & 519.5 & Wyodak-Anderson & Canyon & 13 & 5.91 & 21.65 & 26.31 & 24.26 & ---- \\
\hline 35 & Groves $12-19-4574$ & $1,230.9$ & Wyodak-Anderson & Big George & 20 & 7.56 & 19.42 & 25.55 & 24.77 & 1.29 \\
\hline 36 & PNG CBM 24-1 & $1,266.4$ & ---- & Pawnee & 4 & 5.26 & 17.69 & 22.01 & 34.07 & 1.34 \\
\hline 37 & PNG CBM 26-1 & $1,166.1$ & ---- & Pawnee & 7 & 5.90 & 16.33 & 21.60 & 20.91 & 1.34 \\
\hline
\end{tabular}


Table 2. Properties and composition of coal beds cored-continued

\begin{tabular}{|c|c|c|c|c|c|c|c|c|c|c|c|c|c|c|}
\hline \multirow{2}{*}{$\begin{array}{l}\text { Core } \\
\text { hole }\end{array}$} & \multicolumn{3}{|c|}{$\begin{array}{c}\text { Proximate analysis, in } \\
\text { percent }\end{array}$} & \multicolumn{5}{|c|}{ Ultimate analysis, in percent } & \multirow{2}{*}{$\begin{array}{c}\text { Calorific } \\
\text { value, in } \\
\text { Btu }\end{array}$} & \multirow{2}{*}{$\begin{array}{l}\text { Moist, } \\
\text { mineral- } \\
\text { matter- } \\
\text { free Btu }\end{array}$} & \multirow{2}{*}{$\begin{array}{l}\text { Apparent } \\
\text { rank }\end{array}$} & \multicolumn{3}{|c|}{ Forms of sulfur, in percent } \\
\hline & $\begin{array}{l}\text { Ash } \\
\text { yield }\end{array}$ & $\begin{array}{l}\text { Volatile } \\
\text { matter }\end{array}$ & $\begin{array}{c}\text { Fixed } \\
\text { carbon }\end{array}$ & Hydrogen & Carbon & Nitrogen & Sulfur & Oxygen & & & & Sulfate & Pyritic & Organic \\
\hline 1 & 3.67 & 35.26 & 42.47 & 6.07 & 59.47 & 0.69 & 0.34 & 29.77 & 10,440 & 10,810 & SubA & 0.01 & 0.02 & 0.22 \\
\hline 2 & 3.38 & 34.44 & 43.25 & 6.05 & 59.50 & 0.74 & 0.33 & 30.00 & 10,300 & 10,610 & SubA & 0.03 & 0.07 & 0.24 \\
\hline 3 & 6.30 & 28.44 & 33.56 & 6.43 & 48.78 & 0.54 & 0.22 & 37.75 & 7,790 & 8,690 & SubC & ---- & ---- & ---- \\
\hline 4 & 10.67 & ---- & ---- & 6.14 & 47.55 & 0.78 & 0.22 & 34.66 & ---- & --- & ---- & 0.01 & 0.04 & 0.16 \\
\hline 4 & 5.78 & 33.36 & 38.00 & 6.28 & 53.14 & 0.87 & 0.34 & 33.59 & 9,440 & 9,910 & SubB & 0.01 & 0.10 & 0.23 \\
\hline 5 & 3.95 & 33.03 & 36.65 & 6.54 & 52.33 & 0.59 & 0.22 & 36.36 & 8,920 & 9,300 & SubC & 0.02 & 0.01 & 0.19 \\
\hline 5 & 4.50 & 34.37 & 38.07 & 6.27 & 54.47 & 0.59 & 0.18 & 33.99 & 9,440 & 9,830 & SubB & 0.02 & 0.02 & 0.12 \\
\hline 6 & 5.20 & 27.11 & 33.18 & 6.63 & 47.41 & 0.77 & 0.33 & 39.66 & 7,630 & 8,240 & LigA & 0.02 & 0.10 & 0.22 \\
\hline 6 & 4.54 & 30.53 & 37.48 & 6.38 & 51.39 & 0.85 & 0.14 & 36.70 & 8,670 & 9,190 & SubC & 0.01 & 0.01 & 0.12 \\
\hline 6 & 5.67 & 29.74 & 39.08 & 6.32 & 50.46 & 0.89 & 0.15 & 36.52 & 8,760 & 9,050 & SubC & 0.02 & 0.01 & 0.12 \\
\hline 7 & 14.49 & 28.85 & 27.58 & 6.19 & 39.49 & 0.67 & 0.93 & 38.23 & 7,160 & 8,200 & LigA & 0.12 & 0.26 & 0.55 \\
\hline 8 & 5.87 & 33.08 & 36.46 & 6.22 & 51.27 & 0.85 & 0.35 & 35.45 & 9,080 & 9,550 & SubB & 0.03 & 0.04 & 0.28 \\
\hline 8 & 2.92 & ---- & ---- & 6.22 & 54.45 & 0.72 & 0.10 & 35.58 & ---- & ---- & ---- & 0.04 & 0.01 & 0.06 \\
\hline 8 & 4.19 & 32.38 & 38.59 & 6.25 & 53.21 & 0.82 & 0.27 & 35.26 & 9,340 & 9,750 & SubB & 0.03 & 0.04 & 0.20 \\
\hline 8 & 24.74 & ---- & ---- & 5.05 & 42.55 & 0.67 & 0.72 & 26.27 & ---- & ---- & ---- & 0.05 & 0.27 & 0.40 \\
\hline 9 & 12.22 & 38.51 & 33.60 & 5.99 & 49.18 & 0.75 & 1.05 & 30.82 & 9,280 & 10,160 & SubB & 0.01 & 0.06 & 0.56 \\
\hline 9 & 4.52 & 33.22 & 36.82 & 6.13 & 53.32 & 0.76 & 0.27 & 34.98 & 8,790 & 9,430 & SubC & 0.02 & 0.02 & 0.16 \\
\hline 10 & 9.31 & 32.84 & 34.60 & 6.46 & 48.40 & 0.76 & 0.53 & 34.55 & 8,910 & 9,430 & SubC & 0.02 & 0.05 & 0.47 \\
\hline 10 & 4.59 & 32.15 & 34.84 & 6.59 & 51.56 & 0.76 & 0.29 & 36.21 & 8,750 & 9,840 & SubB & 0.02 & 0.07 & 0.22 \\
\hline 11 & 4.51 & 35.46 & 36.32 & 6.61 & 53.95 & 0.77 & 0.36 & 33.79 & 9,510 & 9,940 & SubB & 0.01 & 0.05 & 0.37 \\
\hline 11 & 2.69 & 30.30 & 41.87 & 6.53 & 56.29 & 0.86 & 0.07 & 33.56 & 9,570 & 9,870 & SubB & 0.01 & 0.01 & 0.06 \\
\hline 12 & 4.58 & 33.11 & 38.19 & 6.30 & 55.99 & 0.70 & 0.34 & 32.09 & 9,300 & 10,100 & SubB & 0.02 & 0.19 & 0.45 \\
\hline 13 & 8.88 & 31.98 & 35.04 & 6.48 & 47.41 & 0.75 & 1.13 & 35.36 & 8,790 & 9,180 & SubC & 0.01 & 0.15 & 0.49 \\
\hline 13 & 4.56 & 32.11 & 35.18 & 6.67 & 50.17 & 0.77 & 0.32 & 37.52 & 8,750 & 9,210 & SubC & 0.01 & 0.01 & 0.20 \\
\hline 14 & 3.46 & 30.92 & 37.37 & 6.69 & 50.62 & 0.63 & 0.23 & 38.36 & 8,890 & 9,210 & SubC & 0.00 & 0.02 & 0.19 \\
\hline 15 & 3.69 & 31.53 & 36.69 & 6.51 & 51.15 & 0.70 & 0.19 & 37.77 & 8,740 & 9,200 & SubC & 0.01 & 0.02 & 0.18 \\
\hline 16 & 4.22 & 31.19 & 36.22 & 6.55 & 51.24 & 0.72 & 0.19 & 37.08 & 8,600 & 9,160 & SubC & 0.01 & 0.07 & 0.21 \\
\hline 17 & 3.82 & 31.24 & 37.78 & 6.58 & 52.30 & 0.70 & 0.20 & 36.40 & 8,770 & 9,120 & SubC & 0.01 & 0.01 & 0.17 \\
\hline 18 & 3.75 & 32.16 & 38.32 & 6.57 & 53.24 & 0.70 & 0.22 & 35.52 & 9,090 & 9,440 & SubC & 0.01 & 0.02 & 0.16 \\
\hline 19 & 3.36 & 34.78 & 38.72 & 6.44 & 54.74 & 0.64 & 0.16 & 34.67 & 9,420 & 9,780 & SubB & ---- & ---- & ---- \\
\hline 20 & 4.50 & 34.27 & 34.93 & 6.60 & 50.38 & 0.68 & 0.35 & 37.46 & 8,990 & 9,510 & SubB & 0.02 & 0.04 & 0.29 \\
\hline
\end{tabular}


Table 2. Properties and composition of coal beds cored-continued

\begin{tabular}{|c|c|c|c|c|c|c|c|c|c|c|c|c|c|c|}
\hline \multirow{2}{*}{$\begin{array}{l}\text { Core } \\
\text { hole }\end{array}$} & \multicolumn{3}{|c|}{$\begin{array}{c}\text { Proximate analysis, in } \\
\text { percent }\end{array}$} & \multicolumn{5}{|c|}{ Ultimate analysis, in percent } & \multirow{2}{*}{$\begin{array}{c}\text { Calorific } \\
\text { value, in } \\
\text { Btu }\end{array}$} & \multirow{2}{*}{$\begin{array}{l}\text { Moist, } \\
\text { mineral- } \\
\text { matter- } \\
\text { free Btu }\end{array}$} & \multirow{2}{*}{$\begin{array}{l}\text { Apparent } \\
\text { rank }\end{array}$} & \multicolumn{3}{|c|}{ Forms of sulfur, in percent } \\
\hline & $\begin{array}{c}\text { Ash } \\
\text { yield }\end{array}$ & $\begin{array}{l}\text { Volatile } \\
\text { matter }\end{array}$ & $\begin{array}{l}\text { Fixed } \\
\text { carbon }\end{array}$ & Hydrogen & Carbon & Nitrogen & Sulfur & Oxygen & & & & Sulfate & Pyritic & Organic \\
\hline 21 & 6.02 & ---- & ---- & 6.77 & 43.14 & 0.63 & 0.88 & 42.57 & ---- & ---- & ---- & 0.07 & 0.26 & 0.55 \\
\hline 21 & 6.91 & 30.73 & 30.53 & 6.70 & 42.56 & 0.70 & 0.51 & 42.62 & 7,590 & 8,030 & LigA & 0.05 & 0.03 & 0.42 \\
\hline 21 & 8.08 & ---- & ---- & 6.62 & 43.00 & 0.76 & 0.67 & 40.87 & ---- & ---- & ---- & 0.02 & 0.05 & 0.60 \\
\hline 22 & 12.78 & 26.66 & 25.24 & 6.34 & 40.20 & 0.67 & 0.85 & 39.16 & 6,460 & 8,180 & LigA & 0.06 & 0.18 & 0.61 \\
\hline 23 & 12.20 & 34.11 & 30.81 & 6.22 & 44.86 & 0.65 & 0.56 & 35.50 & 8,020 & 8,860 & SubC & 0.03 & 0.11 & 0.42 \\
\hline 24 & 8.74 & 31.99 & 45.06 & 5.70 & 58.60 & 1.07 & 0.65 & 25.25 & 10,640 & 11,130 & SubA & 0.02 & 0.29 & 0.34 \\
\hline 25 & 8.14 & 26.64 & 35.05 & 6.30 & 51.18 & 0.76 & 0.38 & 33.23 & 8,120 & 9,580 & SubB & 0.02 & 0.19 & 0.17 \\
\hline 26 & 3.86 & 34.65 & 42.97 & 6.22 & 58.92 & 0.82 & 0.30 & 29.87 & 10,330 & 10,810 & SubA & 0.02 & 0.01 & 0.11 \\
\hline 27 & 4.55 & 30.99 & 37.76 & 6.28 & 55.53 & 0.88 & 0.11 & 32.63 & 9,200 & 10,190 & SubB & 0.02 & 0.00 & 0.09 \\
\hline 27 & 4.77 & 29.31 & 38.89 & 6.32 & 56.24 & 0.91 & 0.23 & 31.53 & 9,100 & 10,090 & SubB & 0.02 & 0.02 & 0.18 \\
\hline 28 & 4.22 & 30.88 & 37.34 & 6.64 & 51.46 & 1.19 & 0.39 & 36.10 & 8,820 & 9,240 & SubC & 0.01 & 0.02 & 0.30 \\
\hline 29 & 9.75 & 32.28 & 39.62 & 6.29 & 52.35 & 1.09 & 0.30 & 30.22 & 9,820 & 10,360 & SubB & 0.01 & 0.06 & 0.23 \\
\hline 30 & 3.74 & 30.01 & 40.14 & 6.41 & 53.82 & 1.29 & 0.26 & 34.48 & 9,120 & 9,640 & SubB & 0.02 & 0.03 & 0.21 \\
\hline 31 & 5.97 & 36.59 & 38.79 & 6.38 & 57.66 & 0.51 & 0.39 & 29.10 & 9,870 & 10,540 & SubA & 0.02 & 0.07 & 0.31 \\
\hline 32 & 3.41 & 34.02 & 43.79 & 6.26 & 61.46 & 1.06 & 0.20 & 27.60 & 10,440 & 10,990 & SubA & ---- & ---- & ---- \\
\hline 33 & 4.22 & 34.25 & 36.97 & 6.69 & 53.10 & 0.68 & 0.21 & 35.11 & 9,180 & 9,640 & SubB & ---- & ---- & ---- \\
\hline 34 & 4.68 & 32.20 & 37.94 & 6.42 & 52.09 & 0.69 & 0.21 & 35.91 & 9,010 & 9,400 & SubC & 0.01 & 0.01 & 0.17 \\
\hline 34 & 3.71 & 30.97 & 39.08 & 6.45 & 52.82 & 0.76 & 0.13 & 36.13 & 9,090 & 9,560 & SubB & 0.02 & 0.00 & 0.16 \\
\hline 35 & 3.65 & 33.53 & 37.94 & 6.48 & 52.71 & 0.55 & 0.20 & 36.41 & 9,150 & 9,700 & SubB & ---- & ---- & ---- \\
\hline 36 & 6.09 & 34.22 & 38.76 & 6.07 & 55.50 & 0.99 & 0.43 & 30.92 & 10,030 & 10,970 & SubA & 0.01 & 0.66 & 0.11 \\
\hline 37 & 5.58 & 31.74 & 41.82 & 5.98 & 56.00 & 0.97 & 0.19 & 31.31 & 9,740 & 10,320 & SubB & 0.02 & 0.01 & 0.08 \\
\hline
\end{tabular}


Table 3. Coal quality data for coal beds in the Fort Union Formation in the Powder River Basin, Wyoming.

[n , number of samples; PRB, Powder River Basin; Btu, British thermal units per pound; MMMF, moist, mineral-matter-free; SubC, subbituminous C; SubB, subbituminous B; SubA, subbituminous A; ----, not determined. Values reported on an as-recieved basis]

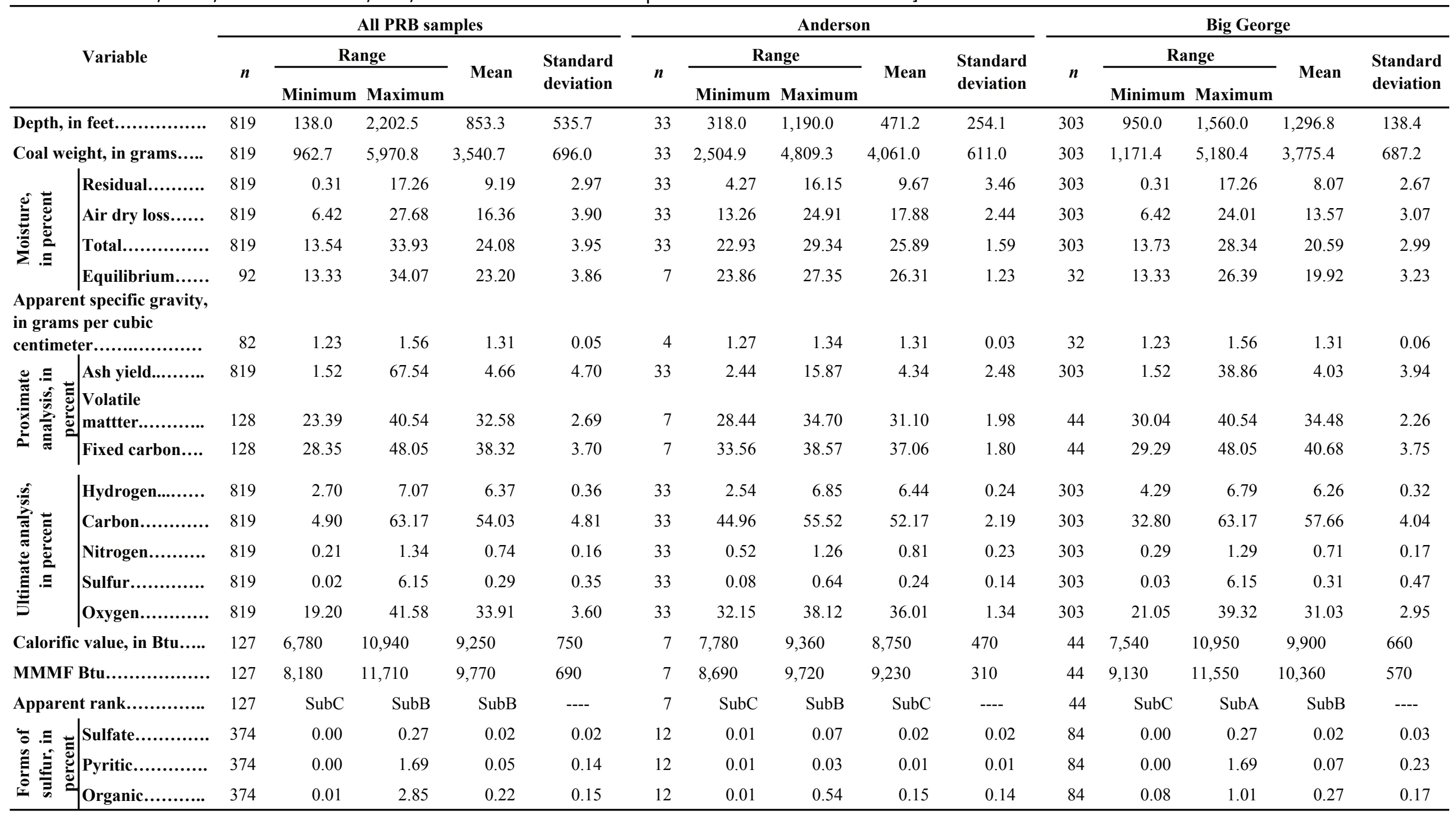


Table 3. Coal quality data-Continued

\begin{tabular}{|c|c|c|c|c|c|c|c|c|c|c|c|c|c|c|c|c|}
\hline \multirow{3}{*}{ 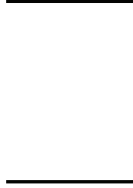 } & \multirow{3}{*}{ Variable } & \multicolumn{5}{|c|}{ Cache } & \multicolumn{5}{|c|}{ Canyon } & \multicolumn{5}{|c|}{ Cook } \\
\hline & & \multirow{2}{*}{$n$} & \multicolumn{2}{|c|}{ Range } & \multirow{2}{*}{ Mean } & \multirow{2}{*}{$\begin{array}{l}\text { Standard } \\
\text { deviation }\end{array}$} & \multirow{2}{*}{$n$} & \multicolumn{2}{|c|}{ Range } & \multirow{2}{*}{ Mean } & \multirow{2}{*}{$\begin{array}{l}\text { Standard } \\
\text { deviation }\end{array}$} & \multirow{2}{*}{$n$} & \multicolumn{2}{|c|}{ Range } & \multirow{2}{*}{ Mean } & \multirow{2}{*}{$\begin{array}{l}\text { Standard } \\
\text { deviation }\end{array}$} \\
\hline & & & Minimum & Maximum & & & & Minimum & Maximum & & & & Minimum & Maximum & & \\
\hline \multicolumn{2}{|c|}{ Depth, in feet................. } & 16 & $1,702.0$ & $1,731.0$ & $1,718.8$ & 9.3 & 31 & 505.5 & 992.0 & 635.6 & 150.6 & 23 & 813.0 & $1,348.0$ & 981.3 & 203.6 \\
\hline \multicolumn{2}{|c|}{ Coal weight, in grams..... } & 16 & $1,320.6$ & $2,458.7$ & $2,037.6$ & 300.3 & 31 & $2,261.0$ & $4,707.2$ & $3,785.9$ & 752.3 & 23 & $2,679.8$ & $5,244.2$ & $3,396.8$ & 578.9 \\
\hline \multirow{4}{*}{ 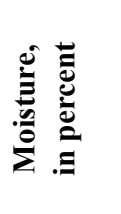 } & |Residual........... & 16 & 4.32 & 14.77 & 9.71 & 2.90 & 31 & 3.72 & 13.54 & 8.16 & 2.82 & 23 & 1.81 & 12.27 & 8.30 & 2.83 \\
\hline & Air dry loss...... & 16 & 9.34 & 20.12 & 14.88 & 2.92 & 31 & 13.77 & 27.68 & 20.63 & 3.67 & 23 & 11.18 & 25.71 & 18.60 & 4.18 \\
\hline & Total............... & 16 & 21.21 & 24.40 & 23.22 & 0.95 & 31 & 23.98 & 33.93 & 27.16 & 2.97 & 23 & 13.54 & 31.02 & 25.36 & 5.27 \\
\hline & Equilibrium...... & 2 & 22.37 & 23.40 & 22.89 & 0.73 & 5 & 24.04 & 26.17 & 25.02 & 0.83 & 0 & ---- & ---- & ---- & ---- \\
\hline \multicolumn{2}{|c|}{$\begin{array}{l}\text { Apparent specific gravity, } \\
\text { in grams per cubic } \\
\text { centimeter................. }\end{array}$} & 2 & 1.26 & 1.28 & 1.27 & 0.01 & 3 & 1.27 & 1.36 & 1.31 & 0.05 & 0 & ---- & ---- & ---- & ---- \\
\hline \multirow{3}{*}{ 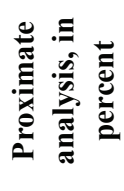 } & Ash yield............ & 16 & 3.33 & 6.19 & 4.03 & 0.71 & 31 & 2.37 & 10.51 & 3.92 & 1.82 & 23 & 2.86 & 67.54 & 11.59 & 16.68 \\
\hline & $\begin{array}{l}\text { Volatile } \\
\text { mattter............. }\end{array}$ & 2 & 28.93 & 29.60 & 29.27 & 0.47 & 9 & 27.03 & 31.92 & 29.73 & 1.80 & 2 & 29.30 & 31.75 & 30.53 & 1.73 \\
\hline & Fixed carbon.... & 2 & 40.74 & 43.22 & 41.98 & 1.75 & 9 & 29.99 & 42.68 & 38.73 & 3.74 & 2 & 36.22 & 38.73 & 37.48 & 1.77 \\
\hline \multirow{5}{*}{ 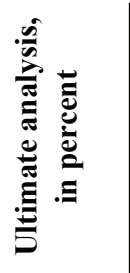 } & |Hydrogen......... & 16 & 6.15 & 6.63 & 6.35 & 0.11 & 31 & 6.08 & 6.76 & 6.46 & 0.16 & 23 & 2.75 & 6.69 & 5.98 & 1.02 \\
\hline & Carbon............. & 16 & 54.38 & 58.34 & 56.78 & 0.89 & 31 & 42.86 & 56.75 & 52.24 & 3.14 & 23 & 10.15 & 57.14 & 47.80 & 10.48 \\
\hline & Nitrogen.......... & 16 & 0.86 & 1.02 & 0.92 & 0.04 & 31 & 0.70 & 1.34 & 0.90 & 0.22 & 23 & 0.21 & 0.95 & 0.78 & 0.16 \\
\hline & Sulfur............. & 16 & 0.07 & 1.09 & 0.23 & 0.26 & 31 & 0.08 & 0.78 & 0.22 & 0.15 & 23 & 0.05 & 1.09 & 0.30 & 0.28 \\
\hline & Oxygen............ & 16 & 29.81 & 32.66 & 31.70 & 0.85 & 31 & 33.25 & 41.49 & 36.27 & 2.34 & 23 & 19.20 & 38.41 & 33.54 & 5.70 \\
\hline \multicolumn{2}{|c|}{ Calorific value, in Btu..... } & 2 & 9,430 & 9,580 & 9,510 & 110 & 9 & 7,250 & 9,750 & 8,890 & 790 & 2 & 8,600 & 8,740 & 8,670 & 100 \\
\hline \multicolumn{2}{|c|}{ MMMF Btu................... } & 2 & 10,040 & 10,110 & 10,080 & 50 & 9 & 8,180 & 10,040 & 9,370 & 660 & 2 & 8,950 & 9,430 & 9,190 & 340 \\
\hline \multicolumn{2}{|c|}{ Apparent rank.............. } & 2 & SubB & SubB & SubB & ---- & 9 & LigA & SubB & SubC & ---- & 2 & SubC & SubC & SubC & ---- \\
\hline \multirow{3}{*}{ 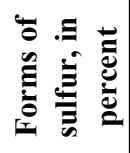 } & Sulfate............. & 16 & 0.00 & 0.05 & 0.02 & 0.02 & 20 & 0.01 & 0.06 & 0.02 & 0.01 & 23 & 0.00 & 0.12 & 0.02 & 0.03 \\
\hline & Pyritic............. & 16 & 0.01 & 0.13 & 0.02 & 0.03 & 20 & 0.00 & 0.21 & 0.05 & 0.06 & 23 & 0.01 & 0.66 & 0.08 & 0.16 \\
\hline & Organic............ & 16 & 0.04 & 0.43 & 0.13 & 0.09 & 20 & 0.06 & 0.52 & 0.19 & 0.12 & 23 & 0.08 & 0.45 & 0.20 & 0.13 \\
\hline
\end{tabular}


Table 3. Coal quality data-Continued

\begin{tabular}{|c|c|c|c|c|c|c|c|c|c|c|c|c|c|c|c|c|}
\hline & \multirow{3}{*}{ Variable } & \multicolumn{5}{|c|}{ Middle / Lower Wyodak } & \multicolumn{5}{|c|}{ Pawnee } & \multicolumn{5}{|c|}{ Roberts } \\
\hline & & \multirow{2}{*}{$n$} & \multicolumn{2}{|c|}{ Range } & \multirow{2}{*}{ Mean } & \multirow{2}{*}{$\begin{array}{l}\text { Standard } \\
\text { deviation }\end{array}$} & \multirow{2}{*}{$n$} & \multicolumn{2}{|c|}{ Range } & \multirow{2}{*}{ Mean } & \multirow{2}{*}{$\begin{array}{l}\text { Standard } \\
\text { deviation }\end{array}$} & \multirow{2}{*}{$n$} & \multicolumn{2}{|c|}{ Range } & \multirow{2}{*}{ Mean } & \multirow{2}{*}{$\begin{array}{l}\text { Standard } \\
\text { deviation }\end{array}$} \\
\hline & & & Minimum & Maximum & & & & Minimum & Maximum & & & & Minimum & Maximum & & \\
\hline \multicolumn{2}{|c|}{ Depth, in feet............... } & 283 & 152.0 & 403.0 & 277.0 & 56.6 & 27 & $1,154.0$ & $1,547.0$ & $1,346.0$ & 165.7 & 7 & $2,188.5$ & $2,200.5$ & $2,196.5$ & 4.3 \\
\hline \multicolumn{2}{|c|}{ Coal weight, in grams..... } & 283 & $2,365.9$ & $5,970.8$ & $3,339.3$ & 374.5 & 27 & 962.7 & $4,089.9$ & $2,331.2$ & 710.1 & 7 & $4,084.5$ & $5,104.5$ & $4,759.5$ & 333.2 \\
\hline \multirow{4}{*}{ 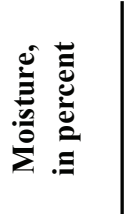 } & |Residual.......... & 283 & 4.77 & 16.90 & 10.26 & 2.66 & 27 & 4.18 & 13.49 & 7.70 & 2.49 & 7 & 3.46 & 6.40 & 5.44 & 1.02 \\
\hline & Air dry loss...... & 283 & 9.89 & 27.04 & 18.59 & 3.29 & 27 & 12.24 & 21.36 & 16.44 & 2.31 & 7 & 15.05 & 21.20 & 18.20 & 2.09 \\
\hline & Total................ & 283 & 17.06 & 33.14 & 26.99 & 2.47 & 27 & 19.09 & 26.85 & 22.99 & 1.84 & 7 & 17.99 & 25.19 & 22.64 & 2.35 \\
\hline & Equilibrium...... & 30 & 22.74 & 28.74 & 25.88 & 1.47 & 6 & 20.41 & 34.07 & 24.17 & 5.16 & 3 & 14.71 & 18.11 & 16.85 & 1.84 \\
\hline \multicolumn{2}{|c|}{$\begin{array}{l}\text { Apparent specific gravity, } \\
\text { in grams per cubic } \\
\text { centimeter.................. }\end{array}$} & 26 & 1.24 & 1.46 & 1.30 & 0.05 & 6 & 1.25 & 1.48 & 1.34 & 0.08 & 3 & 1.28 & 1.38 & 1.34 & 0.05 \\
\hline \multirow{3}{*}{ 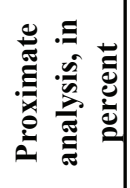 } & Ash yield........... & 283 & 1.92 & 14.58 & 4.11 & 1.67 & 27 & 2.67 & 24.28 & 6.16 & 4.62 & 7 & 3.87 & 38.58 & 9.75 & 12.73 \\
\hline & \begin{tabular}{|l} 
Volatile \\
mattter..............
\end{tabular} & 38 & 28.98 & 37.31 & 31.96 & 1.87 & 6 & 23.39 & 34.22 & 29.94 & 3.63 & 3 & 30.32 & 33.40 & 32.28 & 1.70 \\
\hline & Fixed carbon.... & 38 & 30.41 & 38.61 & 36.25 & 2.03 & 6 & 28.35 & 41.82 & 36.86 & 5.34 & 3 & 39.19 & 39.87 & 39.62 & 0.38 \\
\hline \multirow{5}{*}{ 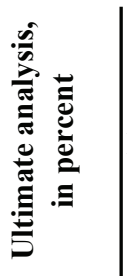 } & Hydrogen.......... & 283 & 5.41 & 7.07 & 6.55 & 0.23 & 27 & 5.54 & 6.63 & 6.16 & 0.29 & 7 & 4.65 & 6.69 & 6.29 & 0.73 \\
\hline & Carbon............. & 283 & 45.07 & 58.21 & 51.58 & 1.87 & 27 & 38.71 & 57.74 & 54.55 & 3.73 & 7 & 31.64 & 57.84 & 52.35 & 9.27 \\
\hline & Nitrogen.......... & 283 & 0.56 & 0.88 & 0.71 & 0.07 & 27 & 0.56 & 1.02 & 0.89 & 0.10 & 7 & 0.71 & 1.23 & 1.09 & 0.17 \\
\hline & Sulfur............. & 283 & 0.03 & 1.43 & 0.25 & 0.17 & 27 & 0.03 & 0.78 & 0.24 & 0.19 & 7 & 0.11 & 0.78 & 0.30 & 0.25 \\
\hline & Oxygen............ & 283 & 30.59 & 41.58 & 36.80 & 1.79 & 27 & 27.42 & 35.21 & 32.01 & 1.83 & 7 & 23.64 & 32.95 & 30.22 & 3.08 \\
\hline \multicolumn{2}{|c|}{ Calorific value, in Btu..... } & 38 & 8,130 & 9,360 & 8,800 & 260 & 6 & 6,780 & 10,030 & 8,920 & 1,210 & 3 & 9,510 & 10,070 & 9,820 & 290 \\
\hline \multicolumn{2}{|c|}{ МMMF Btu................. } & 38 & 8,860 & 10,040 & 9,260 & 250 & 6 & 9,180 & 11,710 & 10,320 & 850 & 3 & 10,110 & 10,620 & 10,360 & 260 \\
\hline \multicolumn{2}{|c|}{ Apparent rank............... } & 38 & SubC & SubB & SubC & ---- & 6 & SubC & SubA & SubB & ---- & 3 & SubB & SubA & SubB & ---- \\
\hline \multirow{3}{*}{ 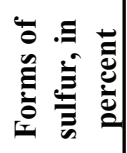 } & Sulfate............. & 131 & 0.00 & 0.13 & 0.01 & 0.02 & 18 & 0.00 & 0.04 & 0.02 & 0.01 & 7 & 0.00 & 0.02 & 0.01 & 0.01 \\
\hline & Pyritic.............. & 131 & 0.00 & 0.27 & 0.03 & 0.05 & 18 & 0.00 & 0.66 & 0.10 & 0.18 & 7 & 0.03 & 0.23 & 0.07 & 0.07 \\
\hline & Organic............ & 131 & 0.06 & 1.06 & 0.22 & 0.15 & 18 & 0.05 & 0.36 & 0.14 & 0.08 & 7 & 0.07 & 0.60 & 0.25 & 0.21 \\
\hline
\end{tabular}


Table 3. Coal quality data-Continued

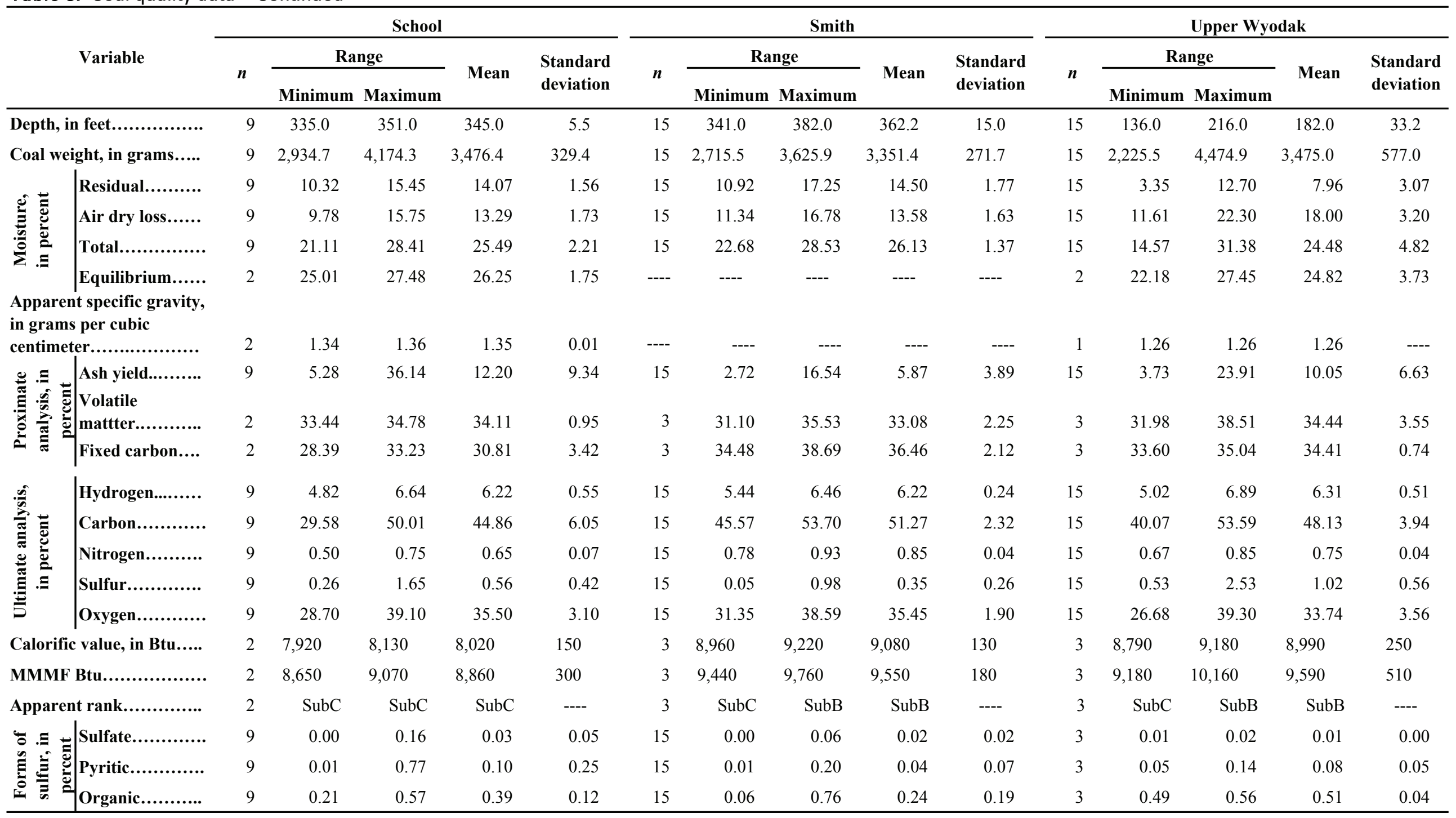


Table 3. Coal quality data-Continued

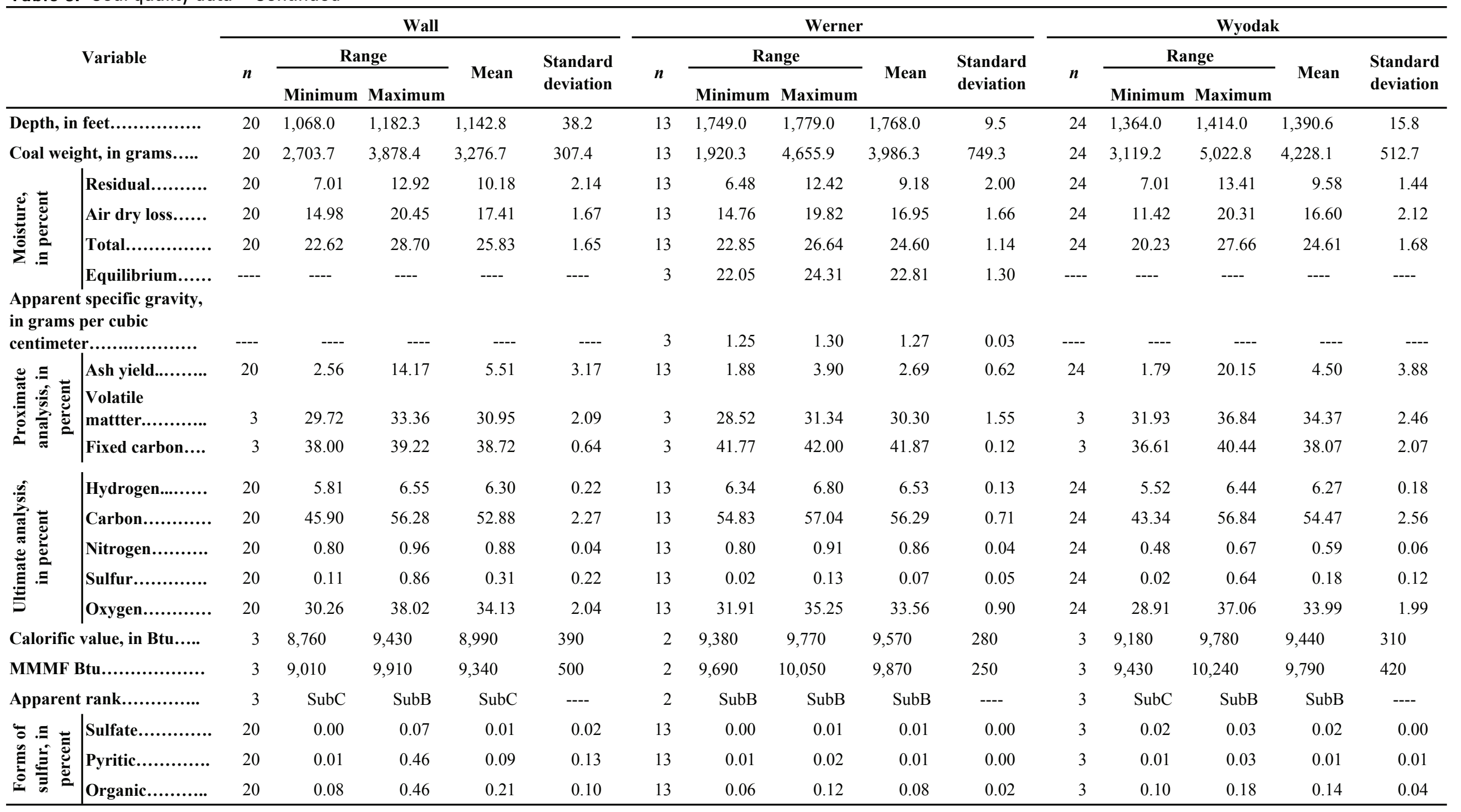


A comparison of the proximate and ultimate analyses (values in mean percent) shows (1) ash yield of the AndersonBig George coal bed ranges from 2.92 to 6.30 percent and that of the Canyon-Wyodak coal bed ranges from 3.46 to 12.22 percent; and (2) total sulfur content for the Anderson-Big George coal bed ranges from 0.10 to 0.39 percent and that for Canyon-Wyodak from 0.13 to 1.13 percent.

Ash yield and total sulfur content within the AndersonBig George coal bed show a generally increasing upward trend interrupted by higher values in the middle part of the bed (for example, see core hole 21; Appendix II), whereas these constituents in the Canyon-Wyodak coal bed show both increasing upward and downward trends locally interrupted by higher values in the middle (for example, see core hole 5; Appendix II). Spikes of higher organic and pyritic forms of sulfur in these beds correspond to local spikes of higher ash yield.

The merged Wyodak coal bed shows generally increasing upward trends for ash yield and total sulfur content (for example, see core hole 14; Appendix II). The merged Big George coal bed shows spikes of moderate to higher ash yield and total sulfur content throughout the coal bed (for example, see core hole 2; Appendix II). Higher organic and sulfate forms of sulfur appear to be directly related to the moderate to higher ash yields.

Variations of MMMF Btu, moisture content, ash yield and total sulfur content were investigated for the WyodakAnderson coal zone in the Wyoming part of the PRB. Figure 6 shows low Btu values $(8,300-9,500)$ in the southern and northwestern parts of the basin, high values $(10,500-11,500)$ in the west-central part of the basin, and intermediate values $(9,500-$ 11,500) elsewhere. Moisture content (fig. 7) shows low values (12-23 percent) in the west-central part of the basin, locally high values (27-35 percent) in the eastern and northeastern parts, and intermediate values (24-26 percent) in other parts. Basinwide variations of ash yield (fig. 8) show high values (10-17 percent) locally in the southern and northern parts of the basin and low values (3.7 percent) in the central part. Total sulfur variations basinwide (fig. 9) show high values (0.5-0.8 percent) in the southern and eastern part of the basin and low values (0.1-0.4 percent) in the central part.

The average ash yields in the Anderson-Big George and Canyon-Wyodak coal beds indicate no significant changes within and between beds, except for local spikes of high ash yield ( $>20$ percent); these higher values may be caused by clay partings introduced by sediment-laden floodwaters or volcanic ash falls. Average sulfur content both within and between these beds also shows no significant difference. Local spikes of moderate to high sulfur are related to thin ash units, especially in the merged Wyodak and Big George coal beds, as exemplified by the presence of as many as 3 ash partings in the merged Wyodak coal bed that can be correlated over a distance of $4 \mathrm{mi}$ (fig. 10). These coal beds, which formed in raised, ombrotrophic mires, were more prone to being inundated by volcanic detritus rather than by floodwaters (Flores, 1986). High ash yield and total sulfur content are commonly correlated to highly degraded maceral types, but, in the merged Wyodak and merged Big George coal beds as indicated above, the partings are mainly formed by volcanic ashfalls. These were nutrient-rich sediments that affected $\mathrm{pH}$ conditions and may have increased plant growth in the mires.

The basinwide variations in MMMF Btu, moisture content, ash yield, and total sulfur content of the WyodakAnderson coal zone are controlled by tectonism, burial history, and depositional environments. The increase in MMMF Btu toward the west-central part of the PRB in Wyoming was caused by tectonic subsidence in which the basin center was downwarped, exposing the strata to higher temperature and pressure. However, the low MMMF Btu of the WyodakAnderson coal zone in the southern part differs from previous reports (Stricker and Ellis, 1999) that calorific values increase toward the southern part of the Gillette coalfield. Thus, the decreasing MMMF Btu toward the southern part of the basin and in the Gillette coalfield indicates a shallower burial history than was originally thought.

\section{Werner Coal Bed}

The Werner coal bed was identified by Kent and others (1980) in the Recluse $1^{\circ}$ x $30^{\prime}$ quadrangle, Wyoming as lying below the Canyon coal bed, where it laterally merges with the Wyodak coal bed toward the south margin of the quadrangle. In the present report, the Werner coal bed was sampled in core hole 11 (see Appendix I, Appendix II). The Werner coal bed is subbituminous B in apparent rank with the following properties and composition:

- Calorific value (mean): 9,570 Btu

- Moist-mineral-matter-free Btu (mean): 9,870

- Proximate analysis (mean percent): ash yield, 2.69; volatile matter, 30.30; fixed carbon, 41.87 ; total moisture, 24.60

- Ultimate analysis (mean percent): hydrogen, 6.53; carbon, 56.29; nitrogen, 0.86; total sulfur, 0.07; oxygen, 33.56

- Forms of sulfur (mean percent): sulfate, 0.004; pyritic, 0.006; organic, 0.058

The vertical variations in ash yield, total sulfur content, and forms of sulfur are displayed in core hole 11 (Appendix II). Ash yield generally decreases upward except for local spikes. Total sulfur content generally increases upward, with random sharp spikes coincident with the higher ash yields. The upward increase of total sulfur is correlated to upward increases of pyritic and organic sulfur.

The upward increasing ash yield and sulfur content of the Werner coal bed may be related to increasing influx of floodwater detritus deposited as partings in the coal in a low-lying swamp. Local spikes of higher ash yield indicate recurring discharges of suspended mud into a swamp, probably during overbank flooding. 


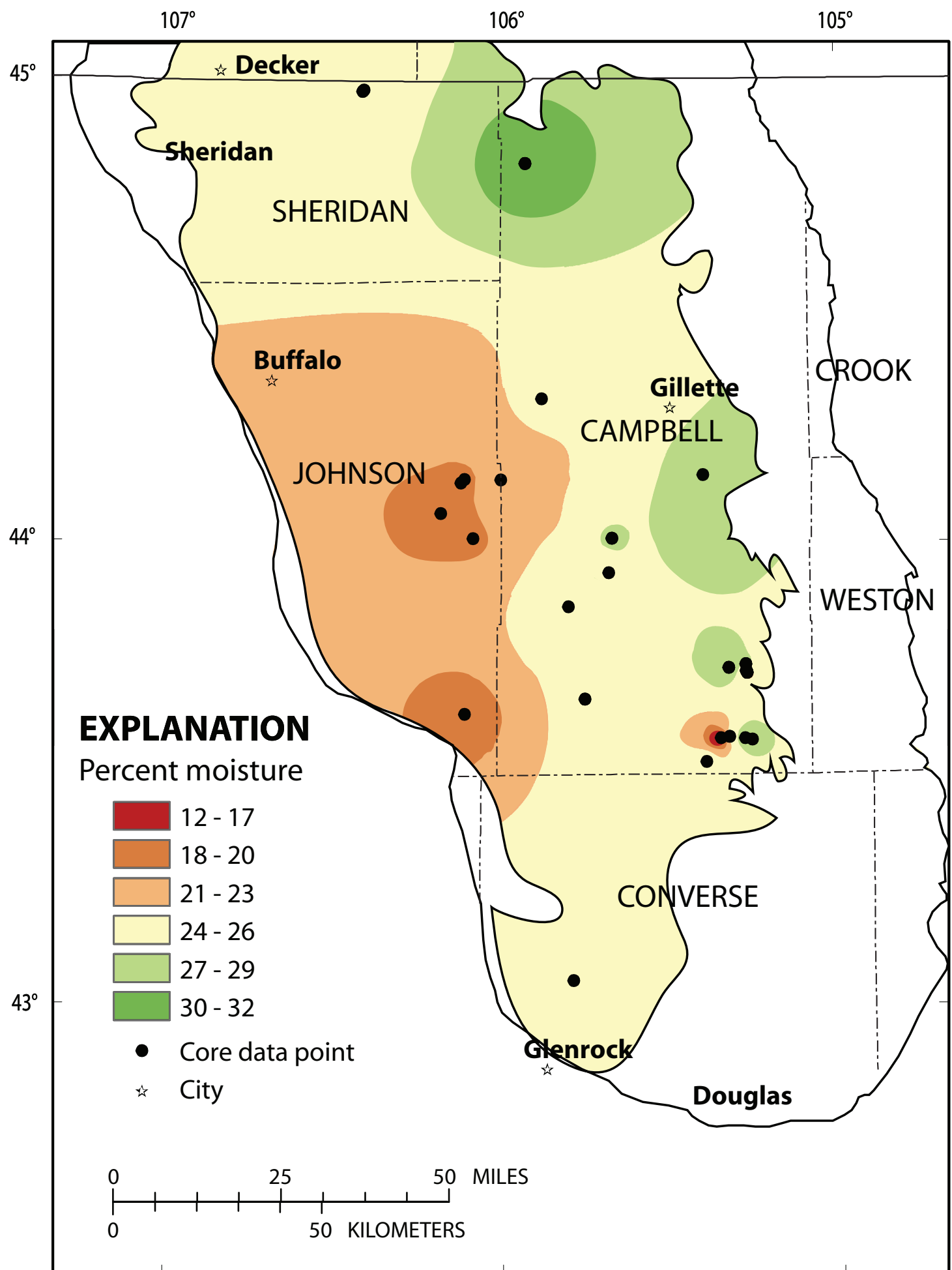

Figure 6. Variation of moist, mineral-matter-free (MMMF) British thermal units (Btu) in the WyodakAnderson coal zone in the Powder River Basin, Wyoming. 


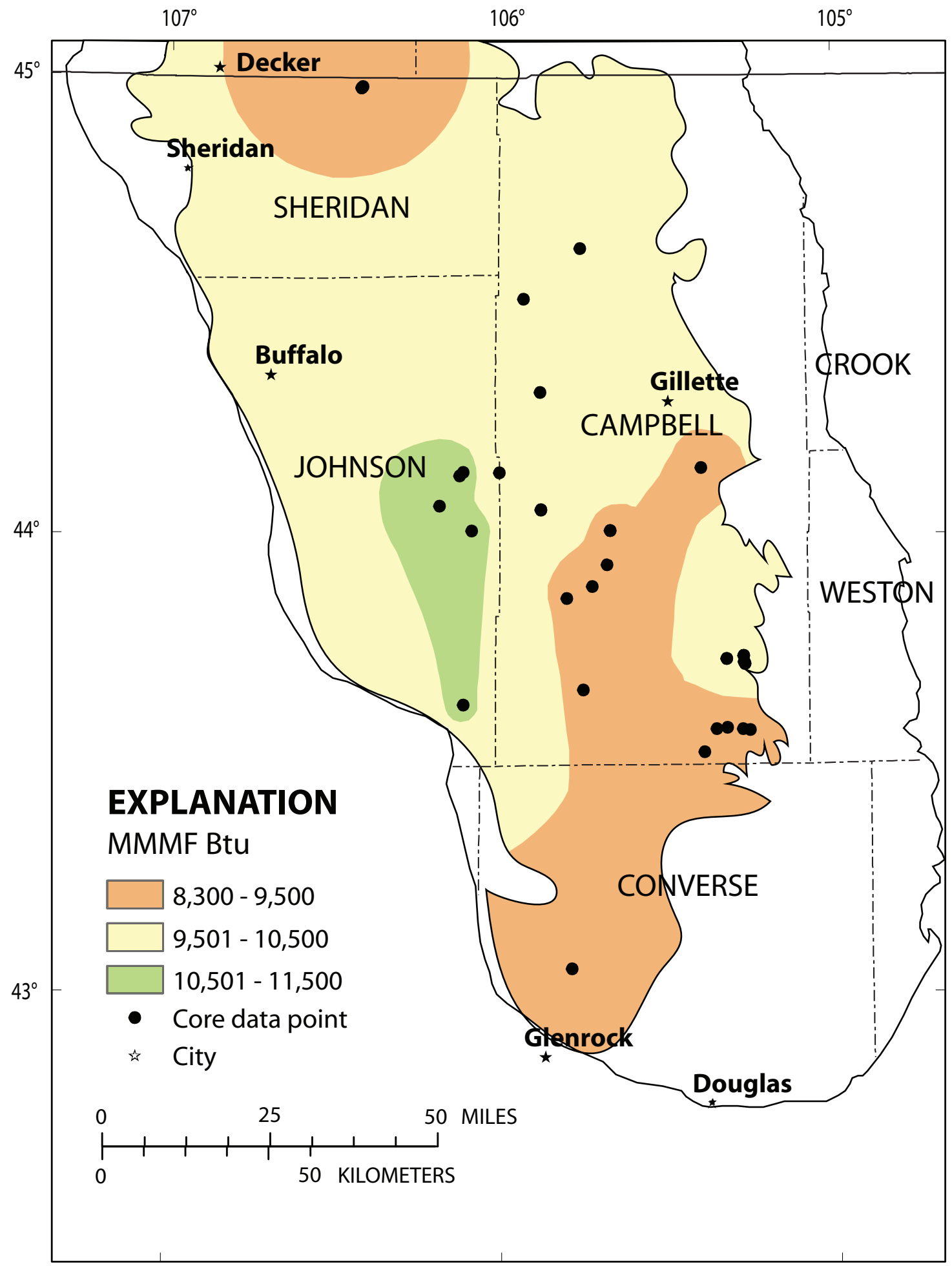

Figure 7. Variation of moisture content in the Wyodak-Anderson coal zone in the Powder River Basin, Wyoming. 


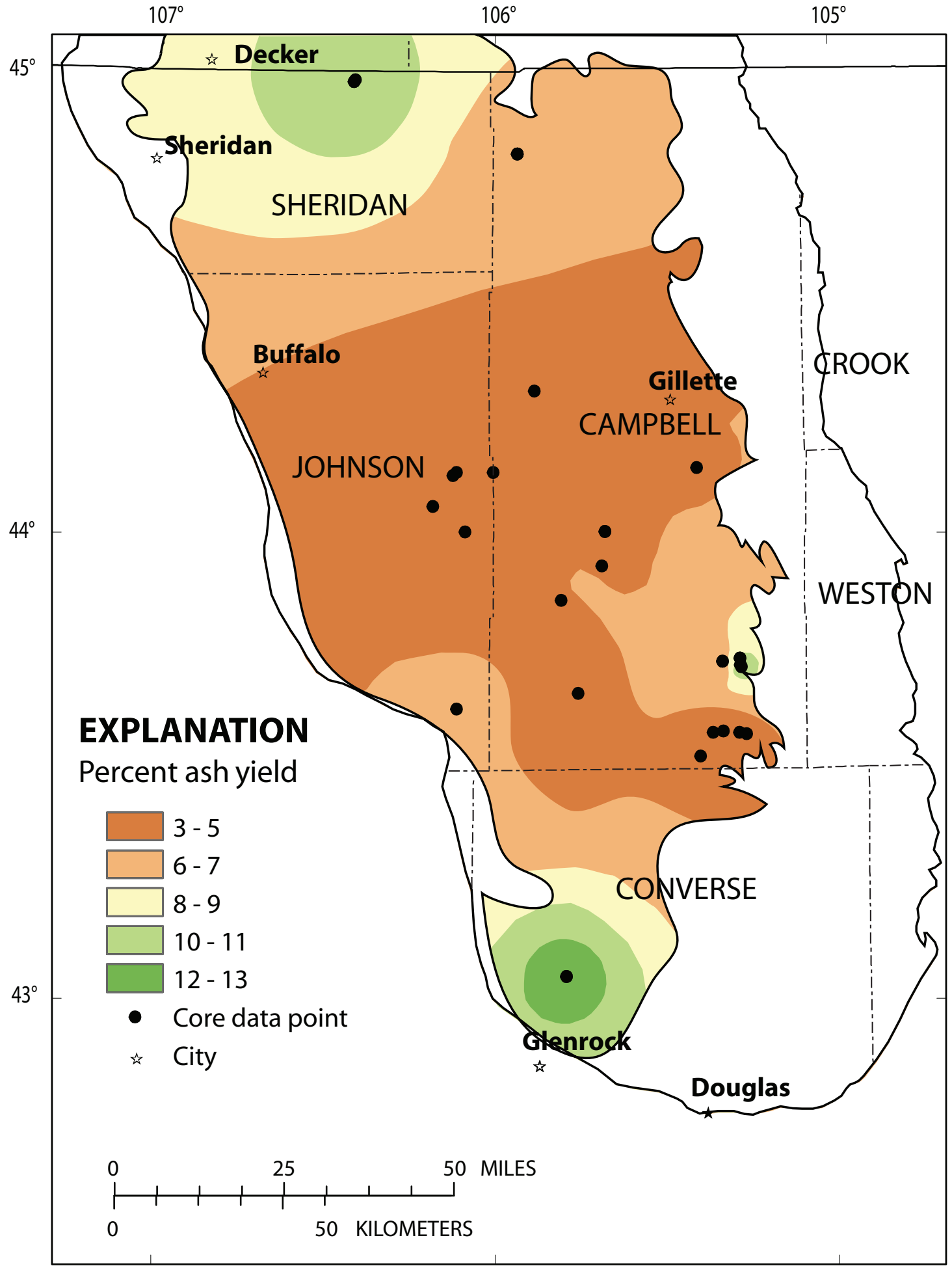

Figure 8. Variation of ash yield in the Wyodak-Anderson coal zone in the Powder River Basin, Wyoming. 


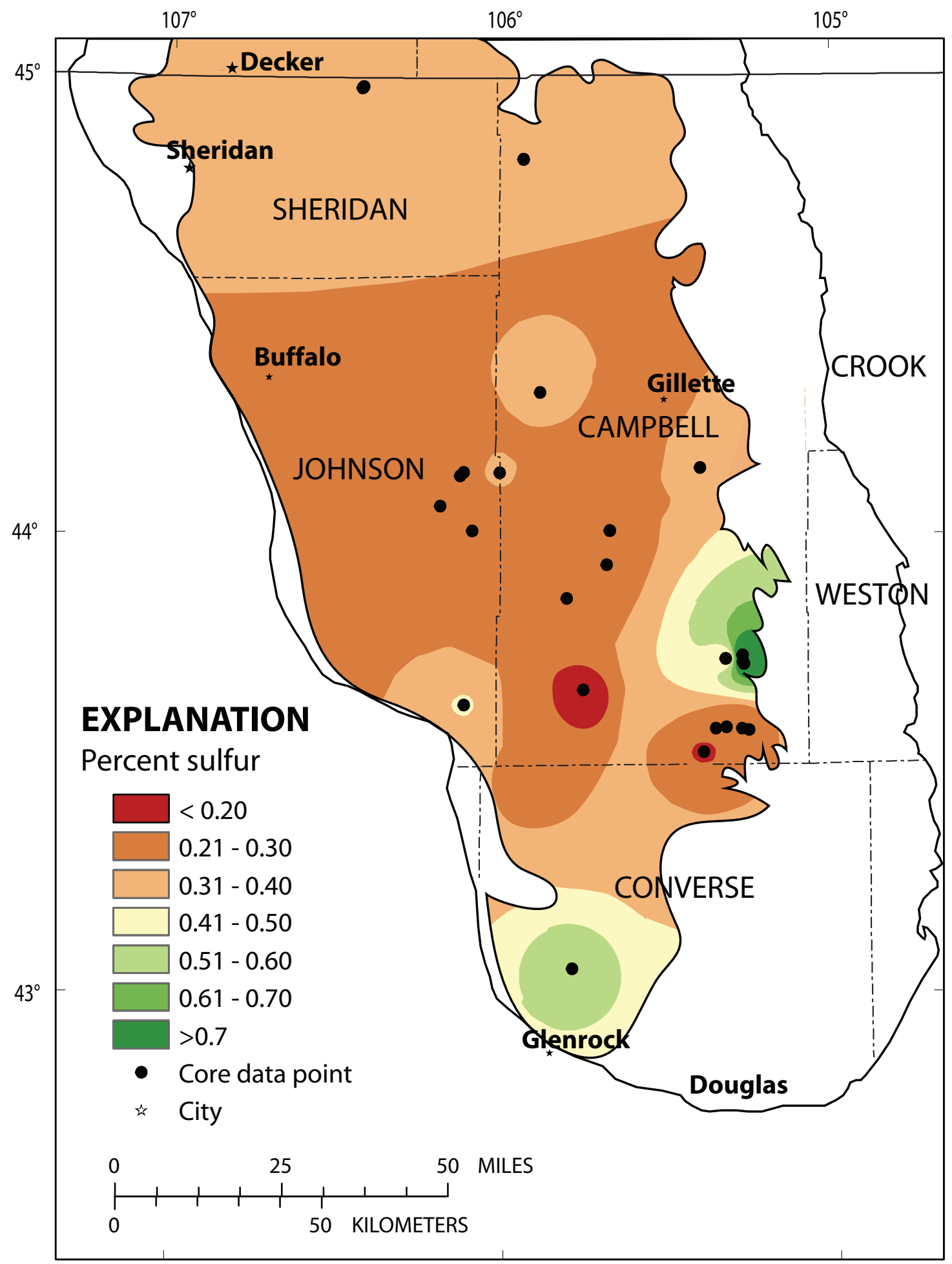

Figure 9. Variation of sulfur content in the Wyodak-Anderson coal zone in the Powder River Basin, Wyoming. 


\section{WEST}

Peabody Natural Gas, LLC.

PNG 35-1

Core hole 18

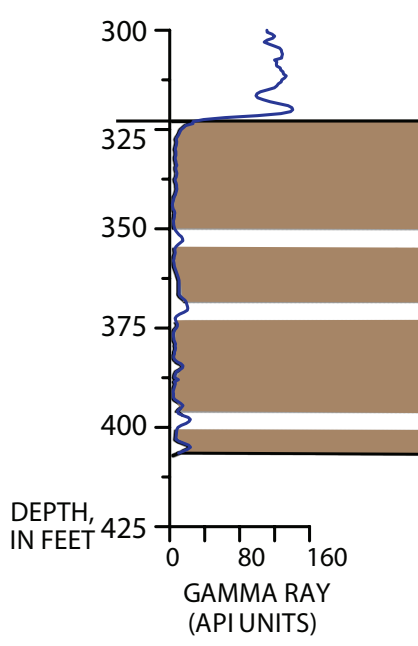

Peabody Natural Gas, LLC.

PNG 31-1 Core hole 17

\section{EAST}

Peabody Natural Gas, LLC. Peabody Natural Gas, LLC. PNG 33-1 Core hole16

PNG 34-1

Core hole 15

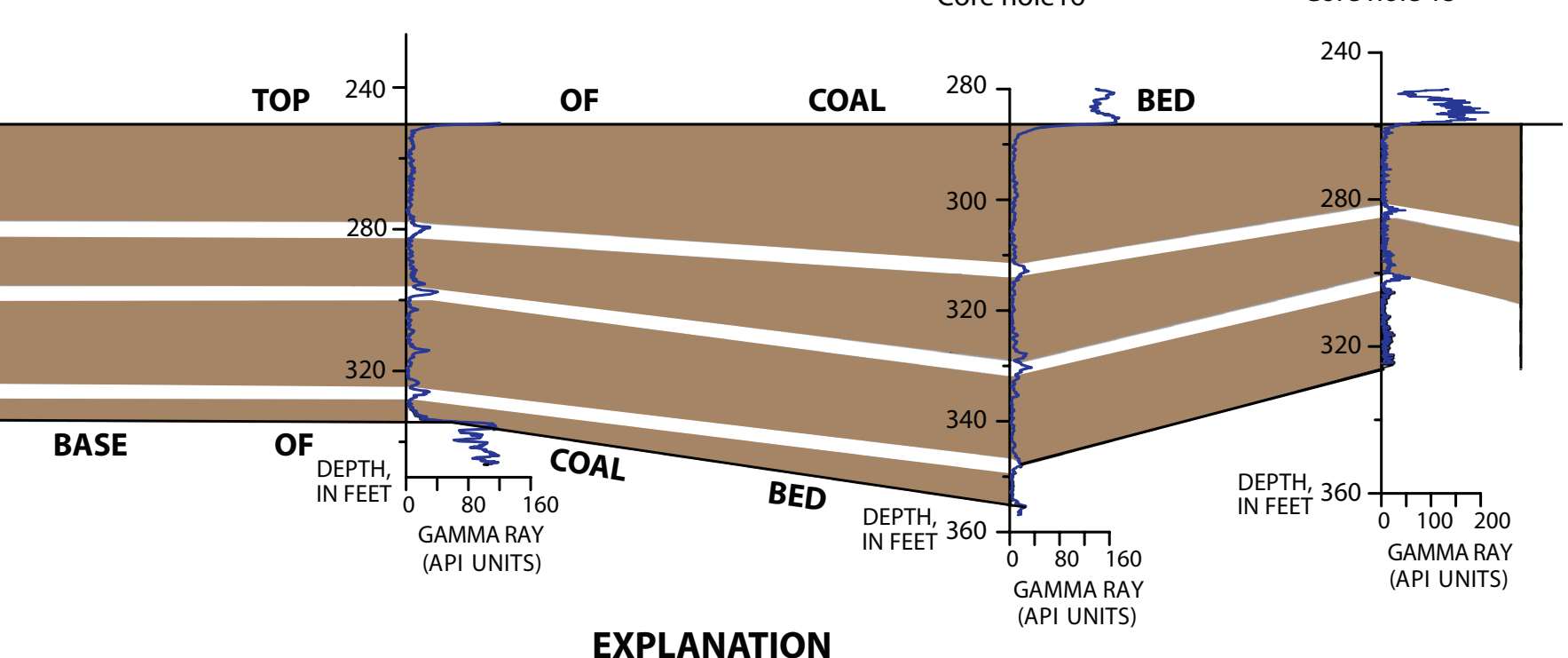

EXPLANATION

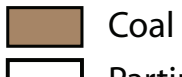

Parting

Figure 10. Ash partings in the merged Wyodak coal bed correlated for core holes 15-18 (fig. 1) in the eastern part of the Powder River Basin, Wyoming. 


\section{Cook Coal Bed}

The Cook coal bed was sampled in core holes 4,6 , and 8 (Appendix I, Appendix II). It is subbituminous C in apparent rank with the following properties and composition:

- Calorific value (mean): 8,670 Btu

- Moist-mineral-matter-free Btu (mean): 9,200

- Proximate analysis (mean percent): ash yield, 11.59; volatile matter, 30.53; fixed carbon, 37.48; total moisture, 25.36

- Ultimate analysis (mean percent): hydrogen, 5.98; carbon, 47.80; nitrogen, 0.78; total sulfur, 0.30; oxygen, 33.54

- Forms of sulfur (mean percent): sulfate, 0.02; pyritic, 0.08 ; organic, 0.20

Vertical variations in the proximate and ultimate analyses are best displayed by ash yield and total sulfur content as related to the sulfate, pyritic, and organic forms of sulfur shown in core holes 4, 6, and 8 (Appendix II). There is a general upward increase in ash yield in core holes 6 and 8, with spikes near the middle of the cores, whereas there is an upward decrease in core hole. The total sulfur content in generally decreases upward in core holes 4 and 8 and increases upward in core hole 6 . The upward decrease of total sulfur in core hole 4 may be related to the upward decrease of pyritic and organic sulfur. However, the upward increase of pyritic and organic sulfur in core hole 6 is directly related to upward increases in pyritic and organic sulfur.

The high ash yield (as high as 24.74 percent) of the Cook coal bed may be related to its depositional setting, which was probably a low-lying swamp inundated by sediment-laden floodwaters. That the swamp was low lying is indicated by spikes of high ash in the middle part of the coal bed as shown in core holes 6 and 8 (Appendix I, Appendix II). The higher total sulfur content (as much as 0.72 percent) may also be related to flooding events.

\section{Wall Coal Bed}

The Wall coal bed was sampled in core hole 4 (Appendix I, Appendix II). The coal is subbituminous B in apparent rank with the following properties and composition:

- Calorific value (mean): 9,440 Btu

- Moist-mineral-matter-free Btu (mean): 9,910

- Proximate analysis (mean percent): ash yield, 5.67; volatile matter, 29.74; fixed carbon, 39.08; total moisture, 27.40

- Ultimate analysis (mean percent): hydrogen, 6.32; carbon, 50.46; nitrogen, 0.89; total sulfur, 0.15; oxygen, 36.52

- Forms of sulfur (mean percent): sulfate, 0.017; pyritic, 0.011 ; organic, 0.117
The vertical variations of the ash yield, total sulfur content, and forms of sulfur in the Wall coal bed are displayed in core hole 4 (Appendix II). The ash yield generally decreases upward, except for a local spike near the base of the coal. The total sulfur content generally increases upward, with random spikes coincident with the spikes of higher ash. The upward increase of total sulfur is correlated to upward increases in pyritic and organic sulfur contents.

The moderate and variable ash yields and upward increasing sulfur content of the Wall coal bed may be related to a more or less constant influx of floodwater detritus into a low-lying swamp. Local spikes of higher ash yield and total sulfur indicate recurring discharges of suspended mud, probably during overbank flooding.

\section{Pawnee Coal Bed}

The Pawnee coal bed was sampled in core holes 25, 27, 36, and 37 (Appendix I, Appendix II). This coal ranges in apparent rank from subbituminous $\mathrm{C}$ to $\mathrm{A}$ with the following properties and composition:

- Calorific value (range of means): 8,120-10,030 Btu

- Moist-mineral-matter-free Btu (range of means): 9,59010,970

- Proximate analysis (range of means in percent): ash yield, 5.58-8.14; volatile matter, 26.64-34.22; fixed carbon, 35.05-41.82; total moisture, 21.60-25.19

- Ultimate analysis (range of means in percent): hydrogen, 5.98-6.30; carbon, 51.18-56.00; nitrogen, 0.760.99 ; total sulfur, $0.19-0.43$; oxygen is $29.87-31.31$

- Forms of sulfur (range of means in percent): sulfate, 0.01-0.02; pyritic, 0.01-0.66; organic, 0.08-0.17

Variable increasing to decreasing upward trends in the ash yield, total sulfur content, and forms of sulfur in the Pawnee coal bed are exhibited in core holes 25, 27, 36, and 37 (Appendix II), which may, in part, be due to the small number of analyzed core samples. In core hole 27 (Appendix II), both ash yield and total sulfur (dominantly organic sulfur) are highest at the top and bottom of the bed.

This trend (in core hole 27) may not be related to flooding events, but rather reflects the normal beginning and ending of the swamp.

\section{Cache Coal Bed}

The Cache coal bed is represented in core hole 27 (Appendix I, Appendix II). Apparent rank is subbituminous B with the following properties and composition:

- Calorific value (mean): 9,100 Btu

- Moist-mineral-matter-free Btu (mean): 10,090

- Proximate analysis (mean percent): ash yield, 4.77; volatile matter, 29.31; fixed carbon, 38.89; total moisture, 23.10 
- Ultimate analysis (mean percent): hydrogen, 6.32; carbon, 56.24; nitrogen, 0.91; total sulfur, 0.23; oxygen, 31.53

- Forms of sulfur (mean percent): sulfate, 0.02; pyritic, 0.02 ; organic, 0.18

The vertical variations of the ash yield, total sulfur content, and forms of sulfur are shown in core hole 27 (Appendix II). Both the ash yield and sulfur content generally decrease upward, with a spike of high sulfur in the uppermost part. The vertical trend for sulfur content follows a general decreasing trend for the pyritic and organic sulfur components.

The mean yield for the Cache coal bed is generally higher than that for the Wyodak-Anderson coal zone ( 3 percent to more than 6 percent), indicating that the original depositional swamp was frequently inundated by floodwaters. Although floodwaters abated through time, spikes of high ash probably reflect intermittent overbank sedimentation into a low-lying swamp. The decreasing upward trend of total sulfur content indicates that there is no relation between sulfur content and overbank flooding. The spike of high sulfur at a depth of $1,710 \mathrm{ft}$ does not appear to be related to any increase in ash yield. However, at the depth of $1,710 \mathrm{ft}$, an increase in ash or possibly a "mud" parting is indicated on the gamma ray log (Stricker and others, 2006; Appendix I-38).

\section{Roberts Coal Bed}

The Roberts coal bed, the lowermost bed sampled in this study of the Fort Union Formation in the PRB, is represented in core hole 29 (Appendix I, Appendix II). The coal is subbituminous $\mathrm{B}$ in apparent rank with the following properties and composition:

- Calorific value (mean): 9,820 Btu

- Moist-mineral-matter-free Btu (mean): 10,360

- Proximate analysis (mean percent): ash yield, 9.75; volatile matter, 32.28; fixed carbon, 39.62; total moisture, 22.64

- Ultimate analysis (mean percent): hydrogen, 6.29; carbon, 52.35; nitrogen, 1.09; total sulfur, 0.30; oxygen, 30.22

- Forms of sulfur (mean percent): sulfate, 0.01; pyritic, 0.06 ; and organic, 0.23

The vertical variations in ash yield, total sulfur content, and forms of sulfur are shown in core hole 29 (Appendix II). The ash yield generally increases upward with a spike (38 percent) at the top of the bed. The total sulfur content gradually increases upward, attaining highest content at the top of the coal bed; organic sulfur content also follows this trend.

The Roberts coal bed, which contains uniform ash yield near 5 percent, except for a spike of 38 percent at the top of the bed, and a total sulfur content that increases upward, is interpreted to have been deposited in a low-lying swamp.
The swamp was frequently inundated by sediment-laden floodwaters, then experienced a peak flooding event that terminated the swamp-like conditions.

\section{Green River Basin}

Six samples were collected from the Deadman 4 coal bed in the Fort Union Formation of the Green River Basin (core hole 24; Appendix I, Appendix II). The coal is subbituminous A in apparent rank with the following properties and composition:

- Calorific value (mean): 10,640 Btu

- Moist-mineral-matter-free Btu (mean): 11,130

- Proximate analysis (mean percent): ash yield, 8.74; volatile matter, 31.99; fixed carbon, 45.06; total moisture, 16.85

- Ultimate analysis (mean percent): hydrogen, 5.70; carbon, 58.60; nitrogen, 1.07; total sulfur, 0.65; oxygen, 25.25

- Forms of sulfur (mean percent): sulfate, 0.02; pyritic, 0.29 ; organic, 0.34

Vertical variations in ash yield, total sulfur content, and forms of sulfur are shown in Appendix II. Ash yield is higher in the middle part of the coal bed than in the lower and upper parts; yields of 12 and 18 percent reflect two ash partings. Total sulfur content generally decreases upward, but attains its highest concentration of more than 0.8 percent where the ash yield is also highest. Pyritic sulfur mimics the total sulfur content, with sulfate and organic forms of sulfur locally high, also corresponding with ash partings.

The Deadman 4 coal bed, which contains high ash yield in the middle part of the bed, is interpreted to have been formed in a low-lying swamp. The sediment-laden floodwaters may have interrupted and drowned peat accumulation twice during the life of the swamp.

\section{Williston Basin}

Seventeen samples were collected from the Beulah coal and 4 unnamed coal beds in the Fort Union Formation in the Williston Basin; these were from core holes 7, 21, and 22 (Appendix I, Appendix II). The coals are lignite A in apparent rank with the following properties and composition:

- Calorific value (range of means): 6,460-7,590 Btu

- Moist-mineral-matter-free Btu (range of means): 8,0308,200

- Proximate analysis (range of means in percent): ash yield, 6.02-14.49; volatile matter, 26.66-30.73; fixed carbon, 25.24-30.53; total moisture, 31.59-34.41

- Ultimate analysis (range of means in percent): hydrogen, 6.19-6.70; carbon, 39.49-43.00; nitrogen, 0.67-0.76; total sulfur, 0.51-0.93; oxygen, 38.23-42.62 
- Forms of sulfur (range of means in percent): sulfate, 0.02-0.12; pyritic, 0.03-0.26; organic, 0.42-0.61

Vertical variations in ash yield, total sulfur content, and forms of sulfur are shown in Appendix II. Ash yield decreases upward in the Beulah coal bed, but increases in the lowermost and uppermost parts of the unnamed coal beds. Sulfur content decreases upward in the Beulah coal bed and also in the unnamed 1 coal bed, but increases upward in the unnamed 2 and the 4 coal beds.

The upward decrease in ash yield in the Beulah coal bed reflects deposition in a swamp that was unaffected by sediment-laden floodwaters. However, for the unnamed coal beds, in which ash yield is, in general, high, flooding events occurred during peat accumulation that was probably a topographically low-lying swamp.

\section{Trace Elements}

Our study of trace elements focused on the merged Big George and merged Wyodak coal beds in the Fort Union Formation of the PRB. Two cores of the Wyodak were obtained, one from core hole 17 (depth $290 \mathrm{ft}$ ) and the other from core hole 18 (depth $370 \mathrm{ft}$ ), and one core of the Big George was collected from core hole 2 (depth 1,100 ft). Although 45 trace elements were analyzed on a whole coal-dry basis (table 4, Appendix III, Appendix IV), primary emphasis was given to 12 trace elements of environmental concern (TEEC) and to comparison of their concentrations both between beds and vertically within beds.

Core holes 17 and 18, from which the two merged Wyodak cores were collected, are located about $1.5 \mathrm{mi}$ apart in the shallow eastern part of the PRB (fig. 1). Except for cadmium, beryllium, and uranium, the majority of the TEEC do not vary significantly between the two core holes in the Wyodak coal bed (see table 4). Mean values for core holes 17 and 18, respectively, for the three elements mentioned are: cadmium, 0.073 and $0.041 \mathrm{ppm}$; beryllium, 0.082 and $0.200 \mathrm{ppm}$; and uranium, 0.58 and $0.46 \mathrm{ppm}$.

Core hole 2 is located in the deep central part of the PRB, 90 mi northwest of core holes 17 and 18 (fig. 1). Comparing the merged Big George coal bed in core hole 2 with the merged Wyodak coal bed in core holes 17 and 18, several of the TEEC vary significantly, as shown by the following (values in mean ppm; Wyodak first, Big George second): arsenic, 1.0 vs. 2.6 ; beryllium, 0.14 vs. 0.31 ; cadmium, 0.057 vs. 0.049 ; manganese, 8.7 vs. 14 ; nickel, 1.3 vs. 2.4 ; selenium, 0.44 vs. 0.97 ; and uranium, 0.52 vs. 0.27 .

Discussion of the vertical variations of TEEC within the merged Wyodak and Big George coal beds includes all of those listed above, except for cadmium. In clay partings (ash content greater than 50 percent) and high ash coal (ash content greater than 15 percent) within these beds, arsenic content is higher than in the beds with lower ash content (less than 15 percent). The Big George, for example, has an arsenic concentration as high as $66 \mathrm{ppm}$ where it contains the highest ash content (13.95 percent, in the depth interval 1,193.5-1,197.5 $\mathrm{ft}$ ), and the Wyodak has arsenic concentrations of 9.2 and 6.0 ppm at its base where the ash content is highest. Beryllium and cadmium increase toward the base and top of the Wyodak coal bed and it decreases upward in the Big George coal bed. Manganese generally increases directly above or below clay partings in the Wyodak coal bed and generally increases upward in the Big George coal bed. Nickel increases toward the base and top of the Wyodak and decreases upward in the Big George. Selenium is invariably high just above or below higher ash yield portions of both coal beds. Uranium is high above and below clay partings and at the base of the Wyodak coal bed and generally increases upward, but is locally variable, above and below clay partings in the Big George coal bed.

In general, the TEEC content of the Wyodak and Big George coal beds, particularly arsenic, beryllium, cadmium, manganese, nickel, selenium, and uranium, is directly related to clay partings or high ash content zones. This indicates that these trace elements are associated either with volcanic ash falls or sediment-laden flood water. The high concentrations at the base and top of the coal beds are due to detrital sedimentation during the early and latter parts of the peat accumulation period. Assuming that these thick coals were deposited in raised bogs (Flores, 1986), detrital sedimentation probably occurred during early development of a low-lying swamp. However, after the swamp evolved into a raised bog, it was seldom drowned by sediment-laden floodwaters. The volcanic ashfalls that created the higher ash zones in these raised bogs are a well-known source of trace elements (Stricker and Flores, 2003).

High contents for TEEC such as arsenic, manganese, nickel, and selenium in the Big George coal bed reflects the proximity of the original depositional swamp to detrital sediments along the western part of the PRB. In addition, proximity to the Elkhorn volcanic arc in southwest Idaho, where the volcanic ash was erupted, accounts for the high TEEC content (Flores and others, 2006). High values for such TEEC as cadmium and uranium in the Wyodak coal bed reflects proximity to provenance to the east and south of the basin (Flores and others, 1994).

\section{Summary}

Fort Union Formation coal beds range from lignite A to subbituminous A in apparent rank. In the Powder River Basin, the apparent rank increases from subbituminous $\mathrm{C}$ in the eastern shallow margins of the basin to subbituminous A in the deeper central part. The minable coal beds (as much as $200 \mathrm{ft}$ thick) in the Wyodak-Anderson coal zone generally have low ash yield and low total sulfur content. The original ash content is either the result of flood events or volcanic ashfalls; the latter is common in coal beds formed on raised ombrotrophic mires. Trace elements of environmental concern are low in the Powder River Basin coals for which major, minor, and trace element contents were determined. 
Table 4. Major, minor, and trace elements in Wyodak and Big George coal beds, Powder River Basin, Wyoming.

[ppm, parts per million; L, means contain less than value(s) (see Appendix III) and were calculated by multipling less than values by 0.5 . All data are on a whole coal dry basis. Number of samples shown in parentheses. Red and blue values are statistically different at the 95 percent level (T-test). ${ }^{*}$, elements of environmental concern]

\begin{tabular}{|c|c|c|c|c|}
\hline \multirow{3}{*}{ Element } & \multicolumn{4}{|c|}{ Core hole number } \\
\hline & $\begin{array}{c}\text { Core holes } 17 \text { and } 18 \\
(79)\end{array}$ & $\begin{array}{c}\text { Core hole } 2 \\
(50)\end{array}$ & $\begin{array}{c}\text { Core hole } 17 \\
\text { (39) }\end{array}$ & $\begin{array}{c}\text { Core hole } 18 \\
(40) \\
\end{array}$ \\
\hline & $\begin{array}{l}\text { Middle/Lower } \\
\text { Wyodak }\end{array}$ & Big George & $\begin{array}{l}\text { Middle/Lower } \\
\text { Wyodak }\end{array}$ & $\begin{array}{c}\text { Middle/Lower } \\
\text { Wyodak }\end{array}$ \\
\hline Aluminum, in percent & 0.48 & 0.40 & 0.45 & 0.50 \\
\hline Calcium, in percent & 0.84 & 0.49 & 0.89 & 0.79 \\
\hline Chlorine, in percent & 0.0079 & $0.0055 \mathrm{~L}$ & $0.0099 \mathrm{~L}$ & $0.0059 \mathrm{~L}$ \\
\hline Iron, in percent & 0.24 & 0.18 & 0.24 & 0.24 \\
\hline Magnesium, in percent & 0.17 & 0.12 & 0.18 & 0.17 \\
\hline Phosphorous, in percent & 0.023 & $0.0058 \mathrm{~L}$ & $0.023 \mathrm{~L}$ & $0.023 \mathrm{~L}$ \\
\hline Potassium, in percent & 0.011 & 0.016 & 0.0092 & 0.013 \\
\hline Silicon, in percent & 0.63 & 0.51 & 0.58 & 0.67 \\
\hline Sodium, in percent & 0.057 & 0.14 & 0.052 & 0.061 \\
\hline Titanium, in percent & 0.045 & 0.040 & 0.045 & 0.045 \\
\hline Antimony, in ppm* & 0.11 & 0.088 & 0.11 & 0.10 \\
\hline Arsenic, in ppm* & 1.0 & 2.6 & 1.1 & 1.0 \\
\hline Barium, in ppm & 450 & 170 & 620 & 290 \\
\hline Beryllium, in ppm* & 0.14 & 0.31 & $0.082 \mathrm{~L}$ & $0.20 \mathrm{~L}$ \\
\hline Bismuth, in ppm & 0.044 & 0.031 & 0.047 & 0.042 \\
\hline Boron, in ppm & 30 & 34 & 28 & 33 \\
\hline Cadmium, in ppm* & 0.057 & $0.049 \mathrm{~L}$ & 0.073 & 0.041 \\
\hline Cesium, in ppm & 0.041 & 0.037 & $0.024 \mathrm{~L}$ & $0.059 \mathrm{~L}$ \\
\hline Chromium, in ppm* & 2.6 & 2.8 & 2.3 & 2.8 \\
\hline Cobalt, in ppm* & 2.0 & 1.8 & 1.9 & 2.0 \\
\hline Copper, in ppm & 9.5 & 9.4 & 9.4 & 9.6 \\
\hline Gallium, in ppm & 1.4 & 1.5 & 1.4 & 1.4 \\
\hline Germanium, in ppm & 0.21 & 0.22 & 0.18 & 0.24 \\
\hline Gold, in ppm & 0.26 & $0.22 \mathrm{~L}$ & $0.27 \mathrm{~L}$ & $0.26 \mathrm{~L}$ \\
\hline Lead, in ppm* & 1.4 & 0.84 & 1.2 & 1.6 \\
\hline Lithium, in ppm & 2.7 & 3.8 & 2.6 & 2.9 \\
\hline Manganese, in ppm* & 8.7 & 14 & 8.1 & 9.4 \\
\hline Mercury, in ppm* & 0.069 & $0.087 \mathrm{~L}$ & $0.063 \mathrm{~L}$ & $0.074 \mathrm{~L}$ \\
\hline Molybdenum, in ppm & 0.37 & $0.35(50$ & 0.39 & 0.34 \\
\hline Neodymium, in ppm & 1.2 & 0.83 & 1.3 & 1.0 \\
\hline Nickel, in ppm* & 1.3 & $2.4 \mathrm{~L}$ & $1.6 \mathrm{~L}$ & $1.0 \mathrm{~L}$ \\
\hline Rubidium, in ppm & 0.53 & 0.60 & 0.40 & 0.67 \\
\hline Scandium, in ppm & 1.1 & 1.4 & 1.0 & $1.2 \mathrm{~L}$ \\
\hline Selenium, in ppm* & 0.44 & 0.97 & $0.45 \mathrm{~L}$ & $0.42 \mathrm{~L}$ \\
\hline Silver, in ppm & 0.52 & $0.029 \mathrm{~L}$ & $0.053 \mathrm{~L}$ & $0.050 \mathrm{~L}$ \\
\hline Strontium, in ppm & 160 & 76 & 150 & 160 \\
\hline Tellurium, in ppm & 0.12 & 0.11 & 0.12 & 0.11 \\
\hline Thallium, in ppm & 0.051 & $0.11 \mathrm{~L}$ & $0.031 \mathrm{~L}$ & 0.072 \\
\hline Thorium, in ppm & 1.3 & $0.69 \mathrm{~L}$ & $1.4 \mathrm{~L}$ & $1.3 \mathrm{~L}$ \\
\hline Tin, in ppm & 0.31 & 0.29 & $0.31 \mathrm{~L}$ & $0.31 \mathrm{~L}$ \\
\hline Uranium, in ppm* & 0.52 & 0.27 & 0.58 & 0.46 \\
\hline Vanadium, in ppm & 10 & 10 & 9.4 & 11 \\
\hline Yttrium, in ppm & 2.2 & 1.7 & $1.8 \mathrm{~L}$ & 2.6 \\
\hline Zinc, in ppm & 13 & 4.9 & 14 & 13 \\
\hline Zirconium, in ppm & 14 & 12 & 13 & 14 \\
\hline Average depth, in feet & 329 & 1,100 & 290 & 370 \\
\hline
\end{tabular}


However, arsenic, beryllium, cadmium, manganese, nickel, selenium, and uranium are higher in the beds with high ash content. Fort Union Formation coal beds in the Green River and Williston Basins are not as low in ash yield and total sulfur content as those in the Powder River Basin. These coals, which are generally thin, formed in low-lying swamps prone to flood events, as evidenced by the clay partings, and were probably deposited along actives areas of fluvial deposition where floods were a common occurrence.

\section{References Cited}

American Society of Testing Materials, 1998: Annual Book of American Society for Testing and Materials Standards, Chapter D 388-98 and D 720-91, p. 174-199.

Bullock, J.H., Cathcart, J.D., and Betterton, W.J., 2002, Analytical methods utilized by the United States Geological Survey for the analysis of coal and coal combustion by-products: U.S. Geological Survey Open-File Report 02-389, 14 p.

Casagrande, D.J., 1979, $\mathrm{H}_{2} \mathrm{~S}$ incorporation in coal precursors: origins of organic sulphur in coal: Nature, v. 282, p. 599-600.

Culbertson, W.C., Kent, B.H., and Mapel, W.J., 1979, Preliminary diagrams showing correlation of coal beds in the Fort Union and Wasatch Formations across the northern Powder River Basin, northeastern Wyoming and southeastern Montana: U.S. Geological Survey Open-File Report 79-1201, 11 p., 2 plates.

Flores, R.M., 1983, Basin facies analysis of coal-rich Tertiary fluvial deposits in the northern Powder River Basin, Montana and Wyoming, in Collinson, J.D., and Lewin, J., eds., Modern and Ancient Fluvial Systems: International Association of Sedimentologists, Special Publication Number 6, p. 501-515.

Flores, R.M., 1986, Styles of coal deposition in Tertiary alluvial deposits, Powder River Basin, Montana and Wyoming, in Lyons, P.C., and Rice, C.L., eds., Paleoenvironmental and Tectonic Controls in Coal-Forming Basins in the United States: Geological Society of America Special Paper 210, p.79-104.

Flores, R.M., Keighin, C.W., Ochs, A.M., Warwick, P.D., Bader, L.R., and Murphy, E.C., 1999a, Framework geology of Fort Union coal in the Williston Basin, North Dakota: A synthesis, in Fort Union Coal Assessment Team, 1999 Resource Assessment of Selected Tertiary Coal Beds and Zones in the Northern Rocky Mountains and Great Plains Region: U.S. Geological Survey Professional Paper 1625-A, Chapter WF, Disc 1, Version 1.0, p. WF-1 - WF-64.

Flores, R.M., Ochs, A.M., and Bader, L.R., 1999b, Framework geology of the Fort Union coal in the eastern Rock Springs uplift, Greater Green River Basin, Wyoming, in Fort Union Coal Assessment Team, 1999 Resource Assessment of
Selected Tertiary Coal Beds and Zones in the Northern Rocky Mountains and Great Plains Region: U.S. Geological Survey Professional Paper 1625-A, Chapter PF, Disc 1, Version 1.0, p. GF-1 - GF-37.

Flores, R.M., Ochs, A.M., Bader, L.R., Johnson, R.C., and Vogler, Daniel, 1999c, Framework geology of the Fort Union coal in the Powder River Basin, in Fort Union Coal Assessment Team, 1999 Resource Assessment of Selected Tertiary Coal Beds and Zones in the Northern Rocky Mountains and Great Plains Region: U.S. Geological Survey Professional Paper 1625-A, Chapter PF, Disc 1, Version 1.0, p. PF-1 - PF-37.

Flores, R.M., and Moore, T.A., 1994, Mechanisms of splitting of the Anderson-Dietz coal bed in the Decker area, Montana; A Synthesis, in Flores, R.M., Mehring, K.T., Jones, R.W., and Beck, T.L., eds., Organics and the Rockies Field Guide: Wyoming State Geological Survey, Public Information Circular No. 33, p. 153-164.

Flores, R.M., McGarry, D.E., and Stricker, G.D., 2005, CBNG development: Confusing coal stratigraphy and gas production in the Powder River Basin: Canadian Society of Petroleum Geologist Gussow Conference, Canmore, Canada, Unpagenated Abstracts with Programs, 1 page.

Flores, R.M., Roberts, S.B., and Perry, W.J., Jr., 1994, Paleocene paleogeography of the Wind River, Bighorn, and Powder River Basins, Wyoming, in Flores, R.M., Mehring, K.T., Jones, R.W., and Beck, T.L., eds., Organics and the Rockies Field Guide: Wyoming State Geological Survey, Public Information Circular No. 33, p. 1-16.

Flores, R.M., Stricker, G.D., Nichols, D.J., 2006, Coal Palynology: Clues to Depositional Environments Controlling Quality and Chemistry of Minable Coals in the Powder River Basin: Geological Society of America Annual Meeting and Exposition, Abstracts and Program, Philadelphia, PA., Oct. 22, 2006, p. 204.

Hettinger, R.D., and Kirschbaum, M.A., 1991, Chart showing correlations of some upper Cretaceous and lower Tertiary rocks, from the east flank of the Washakie Basin to the east flank of the Rocks Springs uplift, Wyoming: U.S. Geological Survey Miscellaneous Investigations Series Map I-2152, 1 sheet.

Kent, B.H., Berlage, L.J., and Boucher, E.M., 1980, Stratigraphic framework of coal beds underlying the western part of the Recluse $1^{\circ}$ x 30' Quadrangle, Campbell County, Wyoming: U.S. Geological Survey Coal Investigations Map C-81, scale 1:100,000.

Kent, B.H., 1986, Evolution of thick coal deposits in the Powder River Basin, northeastern Wyoming, in Lyons, P.C., and Rice, C.L., eds., Paleoenvironmental and tectonic controls in coal-forming basins in the United States: Geological Society of America Special Paper 210, p.105-122. 
Luppens, J.A., Rohrbacher, T.J., Haacke, J.E., Scott, D.C., and Osmonson, L.M., 2006, Status report: USGS coal assessment of the Powder River Basin, Wyoming: U.S. Geological Survey Open-File Report 2006-1072, 30 p.

Mapel, W.J., 1973, Preliminary geologic map of the Rawhide School quadrangle, Campbell County, Wyoming: U.S. Geological Survey Open-File Report 73-177, scale 1:24,000, 1 plate.

McGarry, D.E., and Flores, R.M., 2004, Coalbed natural gas reservoirs Fort Union Formation, Powder River Basin: Coalbed stratigraphy and regional coalbed aquifers: American Association of Petroleum Geology Rocky Mountain Section Meeting, Abstracts with Programs, Unpagenated Abstracts with Programs, 1 page.

Moore, T.A., 1985, Characteristics of coal bed splitting in the Anderson-Dietz coal seam (Paleocene), Powder River Basin, Montana: Unpublished M.S. Thesis, University of Kentucky, 109 p.

Pocknall, D.T., and Flores, R.M., 1987, Coal palynology and sedimentology in the Tongue River Member, Fort Union Formation, Powder River Basin, Wyoming: PALAIOS, v. 2, p. 133-145.

Rohrbacher, T.J., Haacke, J.E., Scott, D.C., Osmonson, L.M., and Luppens, J.A., 2006, Preliminary estimate of coal resources in the Gillette coalfield affected by the location of the Burlington Northern /Union Pacific joint mainline railroad: U.S. Geological Survey Open-File Report 20061206, 16 p.

Sholkovitz, E.R., Boyle, E.A., and Price, N.B., 1978, The removal of dissolved humic acids and iron during estuarine mixing: Earth and Planet, Science Letter, v. 40, p. 130-136.
Staff, Office of the Director of Coal Research, 1967, Methods of analyzing and testing coal and coke: U.S. Bureau of Mines Bulletin 638, 85 p.

Stanton, R.W., 1989, Sampling of coal beds for analysis, in Golightly, D.W., and Simon, F.O., eds., Methods for Sampling and Inorganic Analysis of Coal: U.S. Geological Survey Bulletin 1823, p. 13-23.

Stricker, G.D., and Ellis, M.S., 1999, Chapter PQ-Coal quality and geochemistry, Powder River Basin, Wyoming and Montana, in Fort Union Coal Assessment Team, 1999 Resource Assessment of Selected Tertiary Coal Beds and Zones in the Northern Rocky Mountains and Great Plains Region: U.S. Geological Survey Professional Paper 1625A, Chapter PQ, Disc 1, Version 1.0, p. PQ-1-PQ-24.

Stricker, G.D., and Flores, R.M., 2003, Factors controlling trace elements of environmental concerns for Powder River Basin coal, Wyoming: Geological Society of America Annual Meeting, Seattle, Washington, Abstracts with Programs, v. 35, no. 6, September 2003, p. 229-230.

Stricker, G.D., Flores, R.M., McGarry, D.E., Stilwell, D.P., Hoppe, D.J., Stilwell, K.R., Ochs, A.M., Ellis, M.E., Osvald, K.S., Taylor, S.L., Thorvaldson, M.C., Trippi, M.H., Grose, S.D., Crockett, F.J., and Shariff, A.J., 2006, Gas desorption isotherm studies in coals in the Powder River Basin and adjoining basins in Wyoming and North Dakota: U.S. Geological Survey Open-File Report 2006-1174, 273 p.

Swanson, V.E., and Huffman, Claude, Jr., 1976, Guidelines for sample collecting and analytical methods used in the U.S. Geological Survey for determining chemical composition of coal: U.S. Geological Survey Circular 735, 11 p.

Wood, G.H., Jr., Kehn, T.M., Carter, M.D., and Culbertson, W.C., 1983, Coal resource classification system of the Geological Survey: U.S. Geological Survey Circular 891, 65 p. 